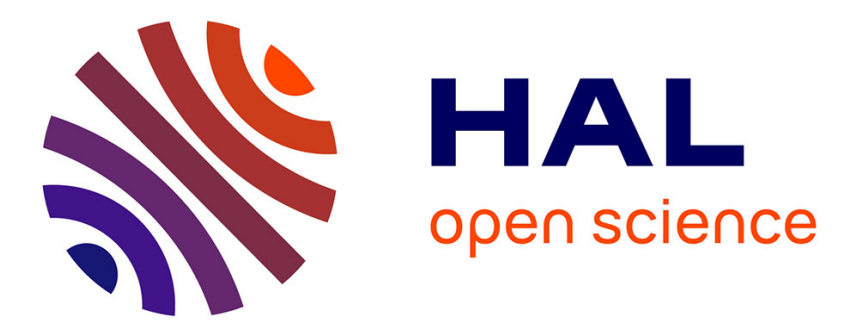

\title{
Sur les champs magnétiques pulsés crowbar. I. Caractéristiques géométriques et électriques des bobines en cuivre
}

S. Askenazy, Ch. Fert, J. Marquez, Ph. Bellan, Ph. Wallace, F. Herlach

\section{- To cite this version:}

S. Askenazy, Ch. Fert, J. Marquez, Ph. Bellan, Ph. Wallace, et al.. Sur les champs magnétiques pulsés crowbar. I. Caractéristiques géométriques et électriques des bobines en cuivre. Revue de Physique Appliquée, 1986, 21 (9), pp.563-577. 10.1051/rphysap:01986002109056300 . jpa-00245474

\section{HAL Id: jpa-00245474 https://hal.science/jpa-00245474}

Submitted on 1 Jan 1986

HAL is a multi-disciplinary open access archive for the deposit and dissemination of scientific research documents, whether they are published or not. The documents may come from teaching and research institutions in France or abroad, or from public or private research centers.
L'archive ouverte pluridisciplinaire HAL, est destinée au dépôt et à la diffusion de documents scientifiques de niveau recherche, publiés ou non, émanant des établissements d'enseignement et de recherche français ou étrangers, des laboratoires publics ou privés. 


\title{
Sur les champs magnétiques pulsés crowbar. I. Caractéristiques géométriques et électriques des bobines en cuivre (*)
}

\author{
S. Askenazy, Ch. Fert, J. Marquez, Ph. Bellan $\left(^{* *}\right)$, Ph. Wallace $\left(^{+}\right)$et F. Herlach $\left(^{++}\right)$ \\ Service CNRS des Champs Magnétiques Intenses et Laboratoire de Physique des Solides, (**) Service de Mathé- \\ matiques, I.N.S.A., Avenue de Rangueil, 31077 Toulouse Cedex, France
}

( $^{+}$McGill University, Montréal, Québec

$\left(^{++}\right)$Katholicke Universiteit, Laboratorium Voor Lage Temperaturen en Hoge-Veldenfysika Celestijnenlaan 200D, B-3030 Leuven, Belgique

(Reçu le 19 juillet 1985, révisé le 27 janvier 1986, accepté le 15 mai 1986)

\begin{abstract}
Résumé. - Un champ pulsé est défini par le champ crête $B_{0}$, l'énergie magnétique $W$ et le rayon intérieur $a_{1}$ des bobines. Nous proposons un ensemble de formules qui, pour chaque couple $w=W / 2,5 a_{1}^{3} B_{0}^{2}$ et $\Gamma=$ diamètre extérieur/longueur des bobines, donnent : l'« épaisseur » de chaque bobine, leur constante de temps, l'homogénéité du champ magnétique, la température du bobinage à la fin d'une impulsion crowbar. Nous montrons, que pour chaque champ pulsé, c'est dans la bobine $\Gamma=1,36$, que les contraintes magnétiques de coeur sont minimales. Nous analysons le rendement magnétique en prenant en compte l'échauffement adiabatique du bobinage. A chaque étape du texte, apparaît l'intérêt des bobines très épaisses.
\end{abstract}

\begin{abstract}
A pulsed magnetic field is characterized by the peak value $B_{0}$, by the magnetic energy $W$ and by the inner coil radius $a_{1}$. We propose a set of formulae giving for any values of $w=W /\left(2.5 a_{1}^{3} B_{0}^{2}\right)$ and $\Gamma$ (outer diameter/ coil length) : the coil « thickness ", the crowbar decay time constant, the field homogeneity, the coil temperature at the end of the magnetic pulse. We show that for any pulsed field, the stress on the inner turns is lowest when choosing the parameter $\Gamma$ equal to 1.36 . We analyse the magnetic field conversion efficiency taking into account the adiabatic heating of the winding. Throughout the paper we emphasize the interest of using very thick coils.
\end{abstract}

\section{Notations.}

C

Capacité du banc de condensateurs.

$V \quad$ Tension de charge.

$B_{0} \quad$ Champ magnétique au centre de la bobine.

$\mu_{0} \quad 4 \pi \times 10^{-7}$

$\mathcal{R} \quad$ Rendement magnétique

$W$ Energie magnétique totale au champ $B_{0} \cdot W=\frac{C V^{2}}{2} \mathcal{R}^{2}$.

$w \quad$ Facteur d'énergie magnétique caractéristique.

$2 a_{1} \quad$ Diamètre intérieur de la bobine.

(*) Ce travail entre dans le cadre du Contrat M.R.T. no $82 \mathrm{G} 0370$.
$2 a_{2} \quad$ Diamètre extérieur.

$2 l$ Longueur de la bobine.

$\Gamma \quad$ Facteur de forme de la bobine. $\Gamma=a_{2} / l$.

$\alpha \quad$ Facteur « d'épaisseur».

$$
\alpha=a_{2} / a_{1}=W^{1 / 3} A(W, \Gamma) .
$$

$\lambda \quad$ Coefficient de remplissage.

D Densité des spires.

$I_{\max }$ Intensité du courant quand le champ est $B_{0}$.

$J$ Densité de courant dans le bobinage à $B_{0} . J=$ $D I_{\max }$.

$L \quad$ Inductance de la bobine. $L=D^{2} a_{1}^{5} \mathfrak{L}(w, \Gamma)$.

$R_{77} \quad$ Résistance de la bobine à l'azote liquide.

T Constante de temps de la bobine à la tempé-

rature de l'azote liquide $\mathfrak{z}=L / R_{77}=a_{1}^{2} \tau(w, \Gamma)$ Temps mis par le champ pour atteindre $B_{0}$. 
$t_{0} \frac{1}{4}$ de période d'un circuit $L, C . \quad t_{0}=$ $\left\{a_{1}^{5} w^{5 / 2} C\right\}^{1 / 2} \frac{D \pi^{2}}{15 \sqrt{5}} t_{0}(w, \Gamma)$

$d_{0}{ }^{1 / 2}$ Paramètre d'amortissement. $d_{0}{ }^{1 / 2}=t_{0} / \pi \tau$.

$\Delta \quad$ Inhomogénéité caractéristique.

$Q \quad$ Densité de chaleur accumulée dans la bobine après un tir.

$Q^{*} \quad Q$ au cour de la bobine, mesuré sous l'effet de la magnétorésistance.

$T(Q)$ et $R(Q)$ Température et résistance de la bobine pour $Q$.

\section{Généralités.}

Il faut savoir que pour de très nombreuses expériences, un champ pulsé quasi-statique à décroissance lente [1a, b] est un excellent substitut aux champs continus.

Il faut savoir que le coût du fonctionnement d'un champ pulsé quasi-statique, à décroissance lente (Fig. 1b), obtenu avec un générateur de type crowbar [2], (Fig. 1a), est insignifiant; quant à l'investissement nécessaire à sa réalisation, il est au moins d'un ordre de grandeur inférieur au prix de construction d'un champ continu intense dont le coût de fonctionnement, en outre, est très important.

D'où l'intérêt des champs pulsés crowbar quasistatiques; ce d'autant plus que les champs réalisés avec cette technique dépassent largement les $30 \mathrm{~T}$ du champ continu actuellement le plus intense [3],

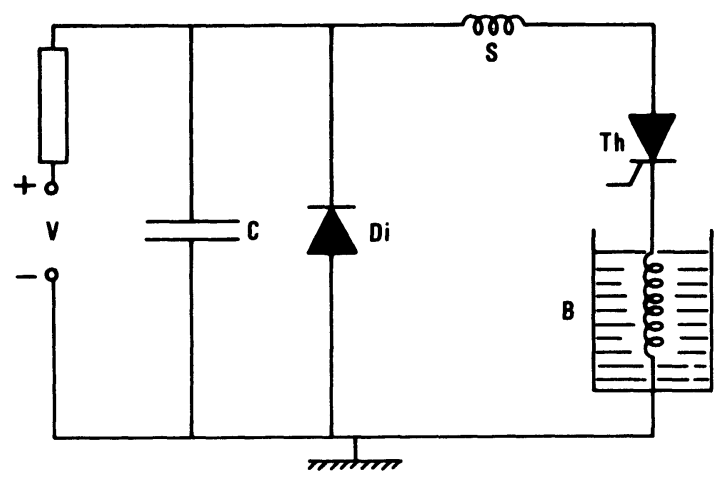

Fig. 1a - Schéma de principe d'un champ pulsé crowbar. Le banc de condensateurs $\mathrm{C}$ est chargé à la tension $V$, puis les thyristors $\mathrm{Th}$ sont amorcés et $\mathrm{C}$ se décharge à travers la bobine B (self $L$, résistance $R_{77}$ ) qui est plongée dans l'azote liquide. Les diodes Di évitent tout retour de l'énergie vers $\mathrm{C}$ (diodes de crowbar). La self de l'inductance de sécurité $S$ est de l'ordre de $L / 100$.

[Schematic diagram of a crowbar pulsed magnet circuit. The storage condenser bank $C$ is charged at the voltage value $V$. The thyristors $T h$ are triggered and enable the discharge of the condensers through the coil B which is immersed in liquide nitrogen (inductance $L$, resistance $R_{77}$ ). The diodes Di prevent the energy feed back to the condensers (crowbar diodes). The safety inductance $S$ is of the order of $L / 100$.]

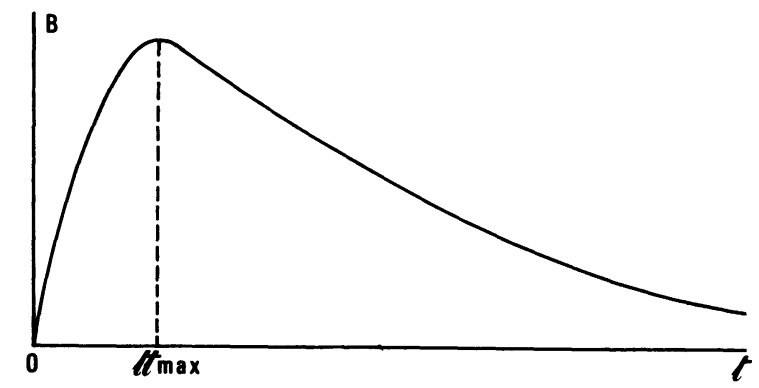

Fig. 1b. - Profil de l'impulsion du champ magnétique. [The magnetic field pulse shape.]

qui est produit depuis 1980 par la bobine hybride du Francis Bitter National Magnet Laboratory.

Citons à titre d'exemple le champ quasi-statique de $45 \mathrm{~T}$ crête que vient de produite le Service CNRS des Champs Magnétiques intenses de Toulouse, en utilisant son banc de condensateurs de 1,25 MJ. L'impulsion de champ(Fig. 1b) est voisine de 1 seconde. La bobine en cuivre est fiable puisque ce n'est qu'après plus de 150 tirs à $45 \mathrm{~T}$ qu'est apparue une légère défaillance $\left({ }^{1}\right)$.

Enfin, il faut retenir qu'un générateur crowbar de $10 \mathrm{MJ}$, permet $50 \mathrm{~T}$ avec une durée d'impulsion de 4 secondes.

Un condensateur est le dual électrique d'une self inductance. Donc stoker l'énergie dans un banc de condensateurs $\left({ }^{2}\right)$ est la solution la plus élégante pour réaliser un générateur de champ crowbar.

Le principe du générateur crowbar est représenté sur la figure 1a. Après que les thyristors, Th, soient amorcés $\left({ }^{3}\right)$, l'énergie $1 / 2 C V^{2}$ du banc $C$ est pour sa plus grande part, transférée sous forme magnétique dans la bobine. Les diodes $D_{i}$, évitent le balancement en retour de l'énergie magnétique en énergie électrostatique dans $C$ (système crowbar). Cela signifie que l'énergie magnétique, après avoir atteint sa valeur maximale à $t^{\max }$ secondes, va se dégrader en chauffant adiabatiquement surtout le bobinage de $B$, car les autres éléments du circuit ont une résistance négligeable.

( $\left.{ }^{1}\right)$ L'utilisation d'un fil de cuivre renforcé préconisée par V. I. Ozhogin et al. [4] augmenterait cette longévité mais ce serait au détriment de la durée de l'impulsion de champ.

$\left({ }^{2}\right) \mathrm{Au}$ prix actuel du $\mathrm{kJ}$ stocké dans un condensateur, au-delà de quelques $\mathrm{MJ}$, il est plus avantageux d'utiliser une machine impulsionnelle [5]. Toutefois le remplacement attendu du traditionnel papier imprégné d'huile, par des films de mylar imprégnés de perfluorocarbone devrait, en réduisant par dix le volume de stockage, abaisser le prix du kilojoule et rendre les condensateurs compétitifs au moins jusqu'à $10 \mathrm{MJ}$.

$\left({ }^{3}\right)$ La référence [6] donne un schéma du déclenchement optique d'un ensemble série-parallèle de thyristors de puissance, qui est adapté à un champ pulsé. 
Ainsi, le courant dans la bobine, donc le champ magnétique, diminuent, figure $1 \mathrm{~b}$, avec une constante de temps qui est au plus égale à celle de la bobine à sa température initiale : $\mathbb{\tau}=L / R_{77}$. C'est la raison du choix d'un bobinage en cuivre refroidi à l'azote liquide. A cette température la résistivité du cuivre est seulement de $0,2 \mu \Omega \mathrm{cm}$ [7], soit plus de 8 fois inférieure à sa résistivité à l'ambiante.

Un calcul élémentaire montre que $\pi^{\max } / \mathbb{\pi}$ sousestime le rapport : énergie joule dissipée au cours du transfert sur énergie totale. Par conséquent, la nécessité d'un rendement magnétique (cf. $\S 8$ ), $R$, de qualité implique $t^{\max } \ll \tau$, ce qui justifie l'intérêt du système crowbar qui après un transfert rapide permet une décroissance lente du champ magnétique.

La conception du générateur de champ est parfaitement simple. Elle n'appelle qu'une seule recommandation : introduire dans le circuit une inductance de sécurité. Son rôle est d'éviter pendant un tir l'apparition accidentelle de courants intempestifs, dommageables et parfois funestes aux diodes et aux thyristors; courants dus par exemple à la brutale mise en courtcircuit, par les forces magnétiques, du bobinage d'une bobine défaillante.

Avant de construire une bobine, il faut choisir ses dimensions. Ce choix est un compromis qui essaie de concilier des données contradictoires qui sont : un budget limité qui fixe l'énergie du champ crowbar, un champ crête fiable le plus intense possible, une grande durée de l'impulsion de champ magnétique et enfin la nécessité d'un volume expérimental confortable au coeur de la bobine.

Notre propos est de donner un ensemble de relations simples qui permettront de déterminer les caractéristiques d'une bobine pour aider ce choix.

La première partie que nous présentons aujourd'hui traite de la dimension des bobines, de leur constante de temps, de leur homogénéité, de leur température en fin de tir; et du rendement magnétique d'un champ crowbar.

Dans la deuxième partie, à venir, nous décrirons les forces magnétiques induites dans le bobinage et leur impact sur la frette qui les contient. Nous arriverons à la conclusion que la distribution des forces magnétiques est minimale dans les bobines pleines, ce qui signifie que les bobines les plus fiables sont des bobines très épaisses.

En définitive tout l'art du champ pulsé crowbar quasi-statique est réduit à la construction de bobines en cuivre convenablement frettées, pour contenir les forces magnétiques induites dans le bobinage, afin que la bobine soit fiable aux champs les plus élevés possibles.

Cette construction implique d'ailleurs plus un savoir faire qu'un savoir.

\section{Introduction.}

Une bobine est définie géométriquement par son rayon intérieur $a_{1}(\mathrm{~cm})$, son rayon extérieur $a_{2}(\mathrm{~cm})$,

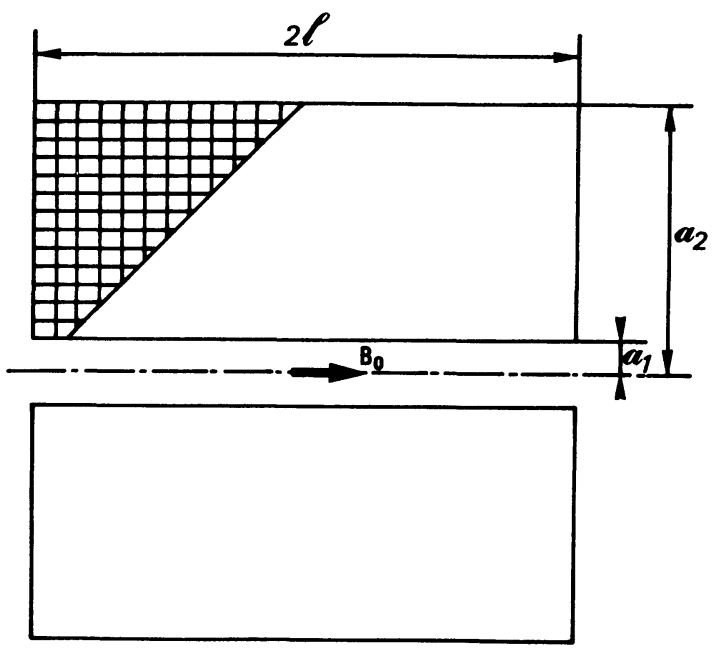

Fig. 2. - Coupe d'une bobine. $\Gamma=a_{2} / l, \alpha=a_{2} / a_{1} ; D$ nombre de spires par $\mathrm{cm}^{2}$.

[A coil section $\Gamma=a_{2} / l, \alpha=a_{2} / a_{1} ; D$ number of turns per $\mathrm{cm}^{2}$.]

sa longueur $2 l(\mathrm{~cm})$ et la densité de bobinage $D$ qui représente le nombre de spires par $\mathrm{cm}^{2}$, figure 2 .

Le rapport $\alpha=a_{2} / a_{1}$ représente l'épaisseur de la bobine. Nous dirons qu'une bobine est épaisse si $\alpha>2$, et avec Butterworth [8] nous désignerons par le terme bobine pleine, celles qui correspondent à $\alpha$ infini.

Le facteur de forme $\Gamma=a_{2} / l$ donne l'aspect extérieur de la bobine. Nous dirons qu'une bobine est longue si $\Gamma<2$, et courte dans l'autre cas.

Les bobines courtes se prêtent mal à un frettage et les bobines trop longues imposent des cryostats trop encombrants. Nous avons dit plus haut que les bobines doivent être épaisses pour être fiables, car nous montrons dans [9] que les contraintes sont des fonctions décroissantes de $\alpha$ qui tendent asymptotiquement vers leur valeur minimale pour $\alpha$ infini, c'est-àdire pour les bobines pleines.

Par conséquent, les bobines pour champs pulsés intenses seront épaisses $\alpha>2$ et relativement peu longues $0,5<\Gamma<2$.

Trois grandeurs définissent un champ pulsé :

- Le diamètre intérieur, $2 a_{1}$ de la bobine.

- Le champ crête $B_{0}$ en son centre.

- L'énergie magnétique globale $W$ au champ crête.

Groupons ces trois grandeurs dans un paramètre $w$, sans dimension, défini par $w=\frac{W}{2 \pi a_{1}^{3} B_{0}^{2} / 2 \mu_{0}}$ que nous désignerons sous le nom de facteur d'énergie magnétique caractéristique et qui définit un champ pulsé.

Le dénominateur $2 \pi a_{1}^{3} B_{0}^{2} / 2 \mu_{0}$, représente l'énergie magnétique au champ crête contenue dans le volume expérimental assimilé à un cylindre de rayon, $a_{1}$, et de longueur $2 a_{1}$. Dans une certaine mesure ce 
dénominateur représente l'énergie magnétique « utile".

Exprimons $a_{1}$ en $\mathrm{cm}, W$ en joules, $B_{0}$ en teslas, alors $w$ prend la forme que nous adopterons dans la suite :

$$
w=\frac{W}{2,5 a_{1}^{3} B_{0}^{2}} .
$$

Le couple $w, \Gamma$ définit une famille de bobines de même " épaisseur » et donc de coefficients caractéristiques identiques et en particulier leur diamètre extérieur, leur constante de temps, leur inductance suivent des règles d'homothétie à partir du rayon $a_{1}$, soit :

$$
\begin{aligned}
& a_{2}=\alpha(w, \Gamma) a_{1} \\
& \widetilde{\tau}=\tau(w, \Gamma) a_{1}^{2} \\
& L=\mathfrak{L}(w, \Gamma) a_{1}^{5} D^{2} .
\end{aligned}
$$

Les réseaux de K. S. W. Champion [10], des courbes iso $S\left(a_{2} / a_{1}, l / a_{1}\right)$, des courbes iso $\phi$ et iso $\lambda$, permettent l'évaluation respective de $\alpha$, $₹$ et $L$.

Nous proposerons une méthode plus simple et en même temps plus précise qui permettra de déterminer aussi bien $\alpha, \tau$ et $\mathcal{L}$ que l'homogénéité du champ dans le cour de la bobine ou sa température en fin de tir. Autre avantage de cette méthode : elle donne une vue d'ensemble de l'évolution des caractéristiques de la bobine en fonction de ses données géométriques.

C'est pourquoi nous pourrons par exemple répondre à la question : quelle est pour chaque champ pulsé $w$, la bobine qui présente en son centre la contrainte magnétique $\sigma_{0}=J B_{0} a_{1}$ [9] minimale ? A l'évidence c'est la bobine qui pour chaque champ pulsé est parcourue par la densité de courant minimale, donc celle qui correspond à $\complement_{w}(\Gamma)$ maximum !
Nous verrons, propriété générale et inattendue des solénoïdes que chacune de ces bobines est définie par le facteur de forme $\Gamma=1,36$.

\section{Détermination de $\alpha(w, \Gamma)$.}

Pour calculer $\alpha(w, \Gamma)$ nous utiliserons l'égalité (1.1) dans laquelle l'énergie magnétique $W$ sera traduite par $W=1 / 2 L I_{\max }^{2}$, ce qui signifie que $B_{0}$ et $L$ sont liés par la relation :

$$
L(\mu \text { henry }) \quad I_{\max }^{2}(\mathrm{Kamp})=5 a_{1}^{3} w B_{0}^{2} .
$$

Nous montrerons que $\alpha=w^{1 / 3} A(w, \Gamma)$ où $A$ est un développement rapidement convergent en puissances de $1 / w^{1 / 3}$.

F. Grover [11] a établi des tables de valeurs numériques de $L\left(a_{1}, a_{2}, l\right)$ définies avec 4 chiffres; où $L$ a été déterminée par une panoplie de formules, chacune valable à l'intérieur de certaines plages de $\Gamma$. Pour les solénoïdes longs et épais la formule la plus propice à nos calculs est le développement de Dwight [12] dont la limite de convergence est $\Gamma=2$.

Introduisons le paramètre $\Gamma$ dans la formule de Dwight et bornons son développement aux termes en $\Gamma^{8}$ tout en y ajoutant le terme correctif $\frac{\Gamma^{9}}{8910} \cdot \frac{13,8-\Gamma}{12}$ pour assurer à cette formule, au moins, la précision des tables de Grover, même à la frontière de $\Gamma=2$, c'est-à-dire jusqu'à $\Gamma=2,05$.

$$
L(\mu \text { henry })=\frac{4 \pi^{2}}{3} \times 10^{-3} D^{2} \frac{\alpha^{5}}{\Gamma} \Lambda a_{1}^{5}
$$

avec pour $\Lambda$ la forme suivante :

$$
\begin{aligned}
& \Lambda=1-4 / \alpha^{3}+3 / \alpha^{4}-\Gamma\left\{0,3661908-1 / \alpha^{3}+0,6416339 / \alpha^{5}+\right. \\
& \left.+\frac{3 \ln \alpha}{20 \alpha^{5}}-\frac{6,696 \times 10^{-3}}{\alpha^{7}}-\frac{8,15 \times 10^{-4}}{\alpha^{9}}-\frac{1,93 \times 10^{-4}}{\alpha^{11}} \ldots\right\} \\
& +\frac{\Gamma^{2}}{12}\left(1+\frac{1}{\alpha^{3}}\right)^{2}-\frac{\Gamma^{4}}{160}\left(1-\frac{1}{\alpha^{3}}\right)\left(1-\frac{1}{\alpha^{5}}\right) \\
& +\frac{\Gamma^{6}}{1792}\left(1-\frac{1}{\alpha^{3}}\right)\left(1-\frac{1}{\alpha^{7}}\right)+\frac{9 \Gamma^{6}}{12800}\left(1-\frac{1}{\alpha^{5}}\right)^{2} \\
& -\frac{5 \Gamma^{8}}{73728}\left(1-\frac{1}{\alpha^{3}}\right)\left(1-\frac{1}{\alpha^{9}}\right)-\frac{9 \Gamma^{8}}{28672}\left(1-\frac{1}{\alpha^{5}}\right)\left(1-\frac{1}{\alpha^{7}}\right) \\
& +\frac{\Gamma^{9}}{8910} \frac{(13,8-\Gamma)}{12} \text {. }
\end{aligned}
$$

Le champ central est donné par

$$
\mu_{0} J l \ln \frac{a_{2}+\sqrt{a_{2}^{2}+l^{2}}}{a_{1}+\sqrt{a_{1}^{2}+l^{2}}}
$$


soit avec les unités adoptées plus haut :

$$
\begin{aligned}
B_{0}=4 \pi \times 10^{-2} D I_{\max }(\mathrm{Kamp}) \frac{\alpha_{1} \alpha}{\Gamma} \ln \times \\
\times \frac{\Gamma+\sqrt{1+\Gamma^{2}}}{\frac{\Gamma}{\alpha}+\sqrt{1+\frac{\Gamma^{2}}{\alpha^{2}}}} .
\end{aligned}
$$

Par conséquent $\alpha$ est solution de l'équation transcendante

$$
\alpha^{3}=\frac{6 w}{\Lambda \Gamma} \ln ^{2} \frac{\Gamma+\sqrt{1+\Gamma^{2}}}{\frac{\Gamma}{\alpha}+\sqrt{1+\frac{\Gamma^{2}}{\alpha^{2}}}}=w A^{3}(\Gamma, \alpha)
$$

Toute calculatrice de poche programmable est à même de résoudre $\alpha(w, \Gamma)$. Toutefois, une méthode graphique s'implifiera la recherche de $\alpha$. Cette méthode est basée sur les remarques qui suivent : (3.4) s'écrit $\alpha=w^{1 / 3} A(\Gamma, \alpha)$; et $A$ peut être développé en série puissance de $1 / \alpha$, donc en série de puissance de $1 / w^{1 / 3}$ puisque $\alpha$ varie surtout comme $w^{1 / 3}$.

$$
A=A_{\infty}\left\{1-\frac{A_{1}}{w^{1 / 3}}-\frac{A_{2}}{w^{2 / 3}} \ldots\right\}
$$

$A_{\infty}$ est la limite de $A$ lorsque $\alpha$ est infini. Par conséquent pour toute la suite, l'indice $\infty$ sera attaché à tous les coefficients qui traduiront le comportement des bobines pleines.

$$
\begin{aligned}
& A^{3}= \frac{6}{\Lambda_{\infty} \Gamma} \ln ^{2}\left(\Gamma+\sqrt{1+\Gamma^{2}}\right) \\
& \Lambda_{\infty}=1-0,3661908 \Gamma+\frac{\Gamma^{2}}{12}-\frac{\Gamma^{4}}{160}+ \\
& \quad+\frac{113 \Gamma^{6}}{89600}-\frac{197 \Gamma^{8}}{516.096}+\frac{(13,8-\Gamma) \Gamma^{9}}{106920} .
\end{aligned}
$$

Quant à $A_{1}$ et $A_{2}$ :

$A_{1}=\frac{2 \Gamma}{z A_{\infty} \ln \left(\Gamma+\sqrt{1+\Gamma^{2}}\right)}$

$A_{2}=\frac{5}{4} A_{1}^{2}$.

Limitons le développement de $A$ au terme $A_{3} / w$ et calculons $A_{3}(\Gamma)$ en écrivant que ce développement ainsi limité, prend exactement la valeur $A(\Gamma, 10)$ pour $w=10$. Alors dès $w>8$, la formule suivante définit $\alpha$, au moins avec 4 chiffres significatifs exacts, ce qui d'ailleurs est superflu !

$$
\alpha=w^{1 / 3} A_{\infty}\left\{1-\frac{A_{1}}{w^{1 / 3}}-1,25 \frac{A_{1}^{2}}{w^{2 / 3}}+\frac{A_{3}}{w}\right\}
$$

$A_{\infty}, A_{1}$ et $A_{3}$ sont représentés sur la figure 3 .

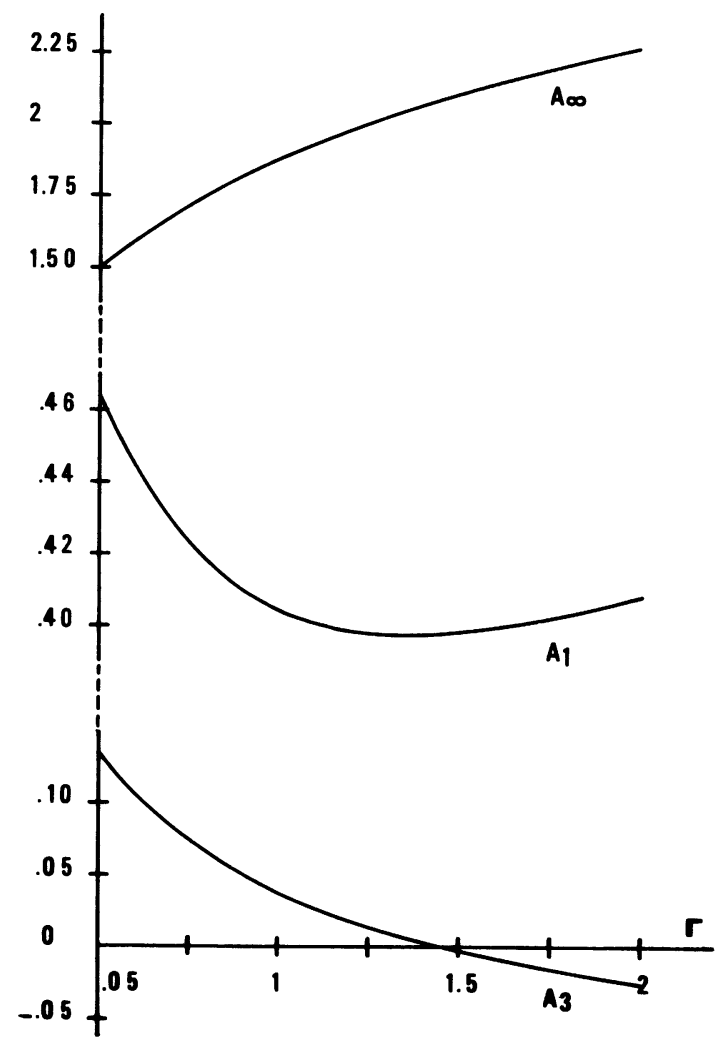

Fig. 3. $-\alpha=w^{1 / 3} A_{\infty}\left\{1-\frac{A_{1}}{w^{1 / 3}}-1,25 \frac{A_{3}}{w^{2 / 3}}+\frac{A_{3}}{w}\right\}$.

Les valeurs numériques de $A_{\infty}, A_{1}$ et $A_{3}$, définies avec 4 chiffres significatifs dans la table I, donnent la possibilité de tracer ces courbes avec précision sur un papier millimétré. Dans ces conditions, la lecture sur ces courbes des valeurs de $A_{\infty}, A_{1}$ et $A_{3}$, définira $\alpha$ à mieux que $0,5 \%$. Compte tenu de cette précision, c'est à partir de $w>5$ que la détermination graphique de $\alpha$ est valable.

\section{La constante de temps $\mathbb{\Psi}$ d'une bobine.}

Ecrivons la résistance du bobinage

$$
R=\rho \frac{\text { volume du bobinage }}{\text { section apparente du conducteur } \times} .
$$

La section vraie du conducteur est égale à $\lambda / D$, où $\lambda$ est le coefficient de remplissage du bobinage

$$
\begin{aligned}
R(\mu \Omega) & =\rho(\mu \Omega . \mathrm{cm}) 2 \pi l\left(a_{2}^{2}-a_{1}^{2}\right) D / \lambda \\
& =2 \pi \rho a_{1}^{3} \frac{D^{2} \alpha^{3}}{\lambda \Gamma}\left(1-\frac{1}{\alpha^{2}}\right) .
\end{aligned}
$$

A partir de l'expression (3.2) qui donne $L$, nous obtenons :

$$
\tau(\mathrm{ms})=\frac{2 \pi \lambda}{3 \rho} a_{1}^{2} \frac{\alpha^{2} \Lambda}{1-\frac{1}{\alpha^{2}}}=a_{1}^{2} \tau
$$


Tableau I. - Valeurs des coefficients qui déterminent $A, \tau, t_{0}, b^{\prime \prime}$ et $\varepsilon$.

[Values of the coefficients, for the determination of $A, \tau, t_{0}, b^{\prime \prime}$ and $\varepsilon$.]

\begin{tabular}{|c|c|c|c|c|c|c|c|c|c|c|c|c|}
\hline$\Gamma$ & $\mathrm{A}_{\infty}$ & $\begin{array}{l}10 \\
A_{1}\end{array}$ & $\begin{array}{l}100 \\
A_{3}\end{array}$ & $\tau_{\infty}$ & $\begin{array}{l}100 \\
\tau_{2}\end{array}$ & $\tau_{3}^{10}$ & $\begin{array}{l}10 \\
t_{3}\end{array}$ & $\begin{array}{c}100 \\
\mathrm{~b}_{\infty}^{\prime \prime}\end{array}$ & $\begin{array}{l}10 \\
b^{\prime \prime}\end{array}$ & $\begin{array}{l}100 \\
\varepsilon_{\infty}\end{array}$ & $\begin{array}{l}10 \\
\varepsilon_{2}\end{array}$ & $\begin{array}{l}10 \\
\varepsilon_{3}\end{array}$ \\
\hline 0,5 & 1,4916 & 4,6440 & 13,44 & 3,9018 & $-12,60$ & 6,972 & 3,396 & 4,177 & 9,774 & 5,995 & 6,112 & 11,16 \\
\hline 0,55 & 1,5409 & 4,5282 & 11,78 & 4,0942 & $-11,36$ & 6,386 & 3,159 & 4,485 & 9,166 & 5,981 & 5,749 & 10,25 \\
\hline 0,6 & 1,5870 & 4,4310 & 10,39 & 4,2701 & $-10,25$ & 5,907 & 2,960 & 4,753 & 8,687 & 5,973 & 5,443 & 9,50 \\
\hline 0,65 & 1,6301 & 4,3492 & 9,18 & 4,4308 & $-9,26$ & 5,508 & 2,788 & 4,983 & 8,308 & 5,971 & 5,182 & 8,86 \\
\hline 0,7 & 1,6706 & 4,2800 & 8,14 & 4,5775 & $-8,35$ & 5,172 & 2,702 & 5,177 & 7,009 & 5,974 & 4,957 & 8,33 \\
\hline 0,75 & 1,7087 & 4,2216 & 7,21 & 4,7112 & $-7,52$ & 4,885 & 2,510 & 5,337 & 7,776 & 5,982 & 4,762 & 7,88 \\
\hline 0,8 & 1,7447 & 4,1723 & 6,39 & 4,8330 & $-6,74$ & 4,638 & 2,397 & 5,466 & 7,598 & 5,994 & 4,591 & 7,49 \\
\hline 0,85 & 1,7787 & 4,1308 & 5,64 & 4,9438 & $-6,01$ & 4,423 & 2,293 & 5,567 & 7,466 & 6,010 & 4,440 & 7,15 \\
\hline 0,9 & 1,8110 & 4,0960 & 4,97 & 5,0444 & $-5,33$ & 4,235 & 2,215 & 5,642 & 7,374 & 6,029 & 4,307 & 6,85 \\
\hline 0,95 & 1,8416 & 4,0671 & 4,35 & 5,1356 & $-4,67$ & 4,068 & 2,142 & 5,696 & 7,316 & 6,052 & 4,189 & 6,60 \\
\hline 1 & 1,8708 & 4,0432 & 3,78 & 5,2181 & $-4,05$ & 3,920 & 2,080 & 5,731 & 7,289 & 6,077 & 4,083 & 6,37 \\
\hline 1,05 & 1,8985 & 4,0239 & 3,25 & 5,2926 & $-3,46$ & 3,787 & 2,028 & 5,749 & 7,288 & 6,105 & 3,989 & 6,17 \\
\hline 1,1 & 1,9250 & 4,0085 & 2,76 & 5,3597 & $-2,88$ & 3,667 & 1,986 & 5,752 & 7,311 & 6,135 & 3,904 & 6,00 \\
\hline 1,15 & 1,9504 & 3,9966 & 2,30 & 5,4201 & $-2,33$ & 3,558 & 1,952 & 5,742 & 7,356 & 6,168 & 3,827 & 5,84 \\
\hline 1,20 & 1,9746 & 3,9878 & 1,87 & 5,4741 & $-1,79$ & 3,458 & 1,926 & 5,723 & 7,424 & 6,202 & 3,757 & 5,71 \\
\hline 1,25 & 1,9978 & 3,9817 & 1,47 & 5,5224 & $-1,27$ & 3,366 & 1,907 & 5,694 & 7,504 & 6,237 & 3,694 & 5,59 \\
\hline 1,30 & 2,0201 & 3,9781 & 1,09 & 5,5653 & $-0,77$ & 3,281 & 1,896 & 5,657 & 7,604 & 6,274 & 3,637 & 5,48 \\
\hline 1,35 & 2,0415 & 3,9767 & 0,73 & 5,6034 & $-0,27$ & 3,202 & 1,891 & 5,615 & 7,719 & 6,313 & 3,585 & 5,39 \\
\hline 1,4 & 2,0621 & 3,9773 & 0,39 & 5,6368 & 0,21 & 3,127 & 1,893 & 5,567 & 7,850 & 6,352 & 3,538 & 5,31 \\
\hline 1,45 & 2,0820 & 3,9796 & 0,06 & 5,6662 & 0,69 & 3,057 & 1,902 & 5,516 & 7,994 & 6,393 & 3,495 & 5,24 \\
\hline 1,5 & 2,1011 & 3,9836 & $-0,25$ & 5,6916 & 1,15 & 2,991 & 1,915 & 5,461 & 8,151 & 6,434 & 3,455 & 5,18 \\
\hline 1,55 & 2,1196 & 3,9889 & $-0,54$ & 5,7135 & 1,61 & 2,933 & 1,936 & 5,403 & 8,320 & 6,477 & 3,419 & 5,13 \\
\hline 1,6 & 2,1374 & 3,9956 & $-0,82$ & 5,7321 & 2,06 & 2,867 & 1,961 & 5,343 & 8,505 & 6,520 & 3,386 & 5,08 \\
\hline 1,65 & 2,1547 & 4,0035 & $-1,08$ & 5,7477 & 2,50 & 2,809 & 1,992 & 5,282 & 8,694 & 6,563 & 3,356 & 5,05 \\
\hline 1,7 & 2,1714 & 4,0124 & $-1,34$ & 5,7605 & 2,94 & 2,752 & 2,027 & 5,220 & 8,893 & 6,607 & 3,328 & 5,02 \\
\hline 1,75 & 2,1876 & 4,0223 & $-1,58$ & 5,7707 & 3,37 & 2,697 & 2,068 & 5,158 & 9,110 & 6,651 & 6,303 & 4,99 \\
\hline 1,8 & 2,2033 & 4,0330 & $-1,81$ & 5,7785 & 3,80 & 2,644 & 2,113 & 5,095 & 9,333 & 6,696 & 3,280 & 4,98 \\
\hline 1,85 & 2,2185 & 4,0446 & $-2,04$ & 5,7841 & 4,22 & 2,591 & 2,162 & 5,032 & 9,566 & 6,741 & 3,259 & 4,96 \\
\hline 1,9 & 2,2334 & 4,0569 & $-2,25$ & 5,7878 & 4,64 & 2,540 & 2,215 & 4,969 & 9,807 & 6,786 & 3,239 & 4,96 \\
\hline 1,95 & 2,2477 & $4,07.00$ & $-2,46$ & 5,7896 & 5,05 & 2,488 & 2,272 & 4,907 & 10,057 & 6,832 & 3,222 & 4,95 \\
\hline 2 & 2,2617 & 4,0837 & $-2,67$ & 5,7899 & 5,46 & 2,438 & 2,331 & 4,845 & 10,316 & 6,879 & 3,206 & 4,95 \\
\hline
\end{tabular}

$\tau$ s'exprime par un développement du même type que celui de $A$, et à $77 \mathrm{~K}$, nous écrivons :

$$
\begin{aligned}
\tau_{77} & =5 w^{2 / 3} \lambda \tau_{\infty}\left\{1-\frac{2 A_{1}}{w^{1 / 3}}-\frac{\tau_{2}}{w^{2 / 3}}-\frac{\tau_{3}}{w}\right\} \\
\tau_{\infty} & =\frac{2 \pi}{3} \Lambda_{\infty} A_{\infty}^{2} \\
\tau^{2} & =1,5 A_{1}^{2}-A_{\infty}^{-2} .
\end{aligned}
$$

La condition de calcul de $\tau_{3}$ est la même que celle de l'évaluation de $A_{3}$ au paragraphe précédent; et la précision du développement proposé, représentatif de $\tau$, est équivalente à celle avancée pour celui de $A$.

Des valeurs de $\tau_{\infty}, \tau_{2}$ et $\tau_{3}$ sont reportées dans la table I et ces fonctions sont représentées sur la figure 4.

$\tau_{\infty}$ croît avec $\Gamma$ et passe d'une valeur de 3,9 pour $\Gamma=0,5$ à une valeur maximale de 5,790 pour $\Gamma=1,98$. Ce maximum est d'ailleurs assez plat puisque à
$\Gamma=1,6, \tau_{\infty}=5,73$. C'est dire, compte tenu du comportement de $A_{1}, \tau_{2}$ et $\tau_{3}$ que pour chaque famille de bobines définie par $w$, toutes celles construites avec $\Gamma$ dans la plage 1,6 à 2 auront la meilleure constante de temps.

Remarquons que $a_{1}^{2} w^{2 / 3}=\left\{W / 2,5 B_{0}^{2}\right\}^{2 / 3}$ est proportionel à la section de la bobine et à $\tau$. Cela signifie que pour avoir une grande constante de temps, il faut une grosse bobine ce qui implique une grande énergie pulsée. Nous retiendrons, comme ordre de grandeur que des bobines de 40 teslas et de $1 \mathrm{~s}$ de constante de temps nécessitent $1 \mathrm{MJ}$ magnétique et un bobinage de 10 litres.

\section{Détermination de la densité de bobinage $D$.}

Le temps d'établissement du champ maximum $t_{\max }$, doit répondre à deux impératifs contradictoires : 


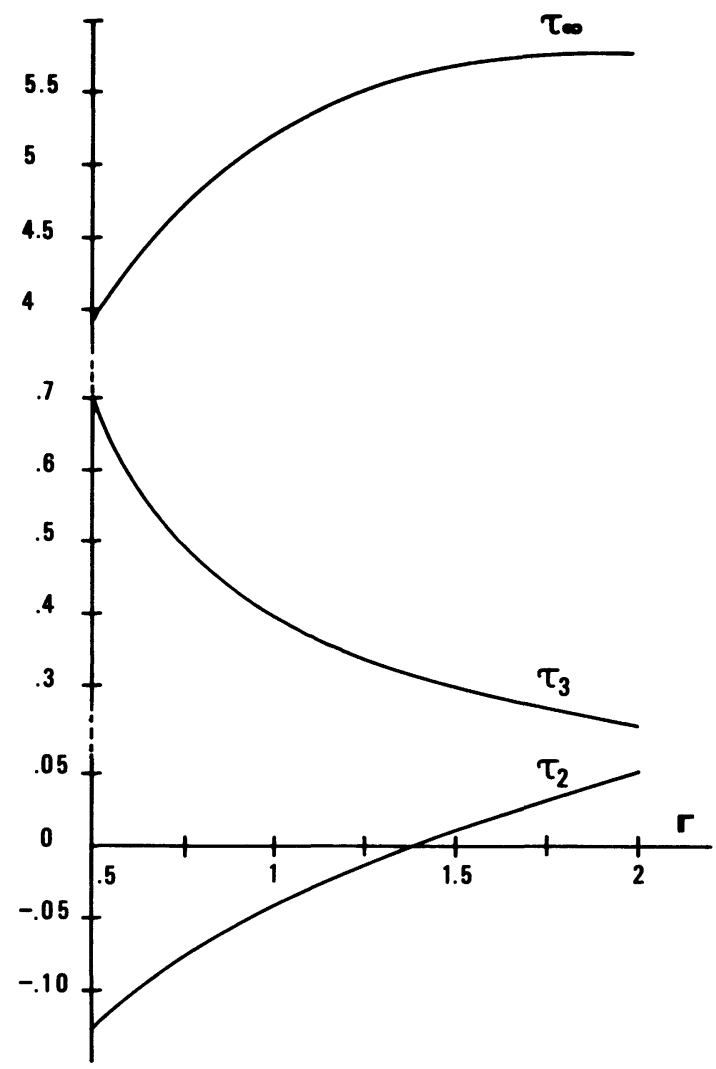

Fig. 4. $-\tau_{77}=5 w^{2 / 3} \lambda \tau_{\infty}\left\{1-2 \frac{A_{1}}{w^{1 / 3}}-\frac{\tau_{2}}{w^{2 / 3}}-\frac{\tau_{3}}{w}\right\}$.

Pour $A_{1}$ : voir figure 3.

[For $A_{1}:$ see figure 3.]

celui d'être le plus long possible pour réduire les effets d'induit et celui d'être d'une durée bien inférieure à $\tau$ bon rendement magnétique.

$t_{0}=\frac{\pi}{2} \sqrt{L C}$ ne surestime que légèrement $t_{\max }$.

Par conséquent, le bon compromis pour $t_{0}$ est de choisir $\pi / 10<t_{0}<\pi / 5$.

Fixer $t_{0}$ est essentiel car il impose $D$, c'est-à-dire qu'il définit la section apparente du fil qui servira à la construction des bobines. $t_{0}$ permet aussi d'estimer le courant qui donne $B_{0}$. Cette estimation, qui est nécessaire pour le choix des composants, est accessible à travers la relation :

$$
I_{\max }(\mathrm{kamp})=\frac{\pi C V \Re}{2 t_{0}(\mathrm{~ms})} .
$$

L'expression (3.2) de $L$ ( $\mu$ henry) peut s'écrire en utilisant (3.4)

$$
L=8 \pi^{2} \times 10^{-3} D^{2} a_{1}^{5} w^{5 / 3} \frac{A^{2}}{\Gamma^{2}} \ln ^{2} \frac{\Gamma+\sqrt{1+\Gamma^{2}}}{\frac{\Gamma}{\alpha}+\sqrt{1+\frac{\Gamma^{2}}{\alpha^{2}}}}
$$

$$
\begin{aligned}
t_{0}(\mathrm{~ms}) & =\frac{\pi}{2} L^{1 / 2}(\mu \text { henry }) C^{1 / 2}(\text { farad }) \\
& =\frac{\pi^{2} D}{15 \sqrt{5}}\left\{a_{1}^{5} w^{5 / 3} C\right\}^{1 / 2} t_{0}
\end{aligned}
$$

La formule suivante qui représente $t_{0}$ est très précise :

$$
t_{0}=A_{1}^{-1}-\frac{2,5}{w^{1 / 3}}-\frac{1,25 A_{1}}{w^{2 / 3}}+\frac{t_{3}}{w} .
$$

Quant à l'inductance, nous l'écrivons :

$L(\mu$ henry $)=D^{2} a_{1}^{5} \mathcal{L} \quad$ avec $\quad \mathcal{L}=\frac{4 \pi^{2}}{1125} w^{5 / 3} t_{0}^{2}$

$t_{3}$ est tracée sur la figure 5 et des valeurs sont reportées sur la table I.

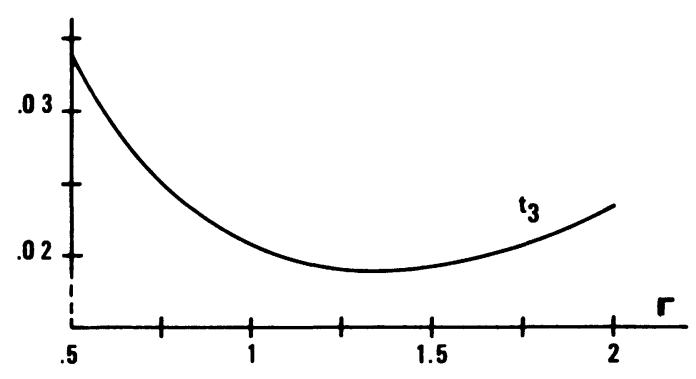

Fig. 5. $-t_{0}=A_{1}^{-1}-\frac{2,5}{w^{1 / 3}}-1,25 \frac{A_{1}}{w^{2 / 3}}+\frac{t_{3}}{w}$.

Pour $A_{1}$ : Voir figure 3.

[For $A_{1}:$ see figure 3.]

$A_{1}$ déjà cité (Eq. (3.6) et Fig. 3) est minimum pour $\Gamma=1,360$ et sa valeur est alors $0,39766 .$. Donc nous en déduisons, vu la forme (5.3) de $t_{0}$, que parmi les bobines définies pour une valeur de $w$, celle qui correspond à $\Gamma=1,360$ présente $\mathcal{L}$ maximum. Cette propriété des solénoïdes est vraie dès que $w>5$.

6. Sur l'homogénéité du champ magnétique dans le volume expérimental.

Il va de soi que le champ au centre d'une bobine est d'autant plus homogène que la bobine est volumineuse et longue. En un point, voisin du centre de la bobine, d'abscisse $z$ et à une distance $r$ de l'axe, le champ qui q une symétrie de révolution, a, en se limitant aux termes d'ordre deux, sa composante parallèle à l'axe [13] qui s'écrit :

$$
B_{z}(z, r)=B_{0}-B_{0}^{\prime \prime}\left(\frac{z^{2}}{2}-\frac{r^{2}}{4}\right)+\cdots
$$

avec $B_{0}^{\prime \prime}=-\frac{\mathrm{d}^{2} B_{z}}{\mathrm{~d} z^{2}}$ au centre de la bobine.

Nous appellerons inhomogénéité caractéristique $\Delta$, l'inhomogénéité maximale dans le volume expérimental, c'est-à-dire : 


$$
\Delta=\frac{B_{0}-B_{z}\left(a_{1}, 0\right)}{B_{0}} \simeq \frac{a_{1}^{2} B_{0}^{\prime \prime}}{2 B_{0}} .
$$

Cela signifie, compte tenu de (6.1), que l'inhomogénéité dans une petite sphère de rayon $a$, centrée dans la bobine est pratiquement égale à $\Delta a^{2} / a_{1}^{2}$.

En général, les auteurs écrivent $B_{z}$ sur l'axe et le comparent à $B_{0}$ pour calculer l'inhomogénéité.

$$
\begin{aligned}
B_{z}=\frac{\mu_{0} J}{2}\left\{(l+z) \ln \frac{a_{2}+\sqrt{(l+z)^{2}+a_{2}^{2}}}{a_{1}+\sqrt{(l+z)^{2}+a_{1}^{2}}}\right. \\
+ \text { idem en }(l-z)\} .
\end{aligned}
$$

Pour obtenir $\Delta$, dérivons deux fois $B_{z}$. La forme brute de $B_{z}^{\prime \prime}$ est lourde même pour $z$ nul [10], comme le montre l'expression suivante transcrite en $\Gamma$ et $\alpha$.

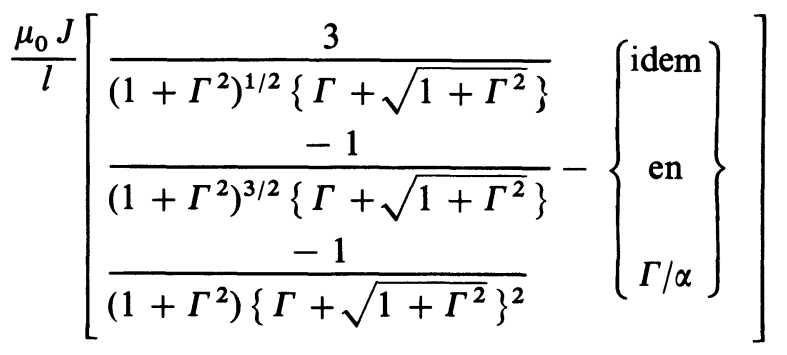

Toutefois, nous remarquons que :

$$
\frac{3}{\left(1+x^{2}\right)^{1 / 2}\left\{x+\sqrt{1+x^{2}}\right\}}-\frac{1}{\left(1+x^{2}\right)^{3 / 2}\left\{x+\sqrt{1+x^{2}}\right\}}-\frac{1}{\left(1+x^{2}\right)\left\{x+\sqrt{1+x^{2}}\right\}^{2}}
$$

se simplifie pour donner $1-\frac{x^{3}}{\left(1+x^{2}\right)^{3 / 2}}$. Ainsi

$$
B_{0}^{\prime \prime}=\frac{\mu_{0} J}{l}\left\{\frac{\Gamma^{3}}{\left(1+\Gamma^{2}\right)^{3 / 2}}-\text { idem en } \Gamma / \alpha\right\} .
$$

P. B. Montgomery [16] obtient un résultat de la même forme à partir du principe de superposition c'est-à-dire en intégrant sur tout le volume du solénoïde, la dérivée seconde axiale du champ d'une spire élémentaire.

En fait (6.3) peut être obtenu simplement en remarquant d'abord que pour $z$ nul $\mathrm{d}^{2} B / \mathrm{dz}^{2}=\mathrm{d}^{2} B /$ $\mathrm{d} l^{2}$, et ensuite que dans (3.3a) $B_{0}=B_{02}-B_{01}$ où $B_{0 i}=\mu_{0} J l\left\{\ln \left(a_{i}+\sqrt{a_{i}^{2}+l^{2}}\right)-\ln l\right\}$ est le champ au centre d'une bobine pleine de rayon extérieur $a_{i}$.

Puisque $B_{0}=\mu_{0} J l \ln \frac{\Gamma+\sqrt{1+\Gamma^{2}}}{\Gamma / \alpha+\sqrt{1+\Gamma^{2} / \alpha^{2}}}$, nous écrirons :

$$
\Delta=\frac{\Gamma^{2}}{2 \alpha^{2}} \frac{\Gamma^{3} /\left(1+\Gamma^{2}\right)^{3 / 2}-\text { idem en } \Gamma / \alpha}{\ln \left(\Gamma+\sqrt{1+\Gamma^{2}}\right)-\text { idem en } \Gamma / \alpha} .
$$

Apprécier $\Delta$ à $1 \%$ près est plus que suffisant.

Le développement proposé ci-dessous fait mieux :

$$
\Delta=\frac{\Gamma^{2}}{w^{2 / 3}} \frac{b_{\infty}^{\prime \prime}}{1-3,5 \frac{A_{1}}{w^{1 / 3}}+\frac{b_{3}^{\prime \prime}}{w}}
$$

$b_{\infty}^{\prime \prime}$ et $b_{3}^{\prime \prime}$ apparaissent dans la figure 6 et la table I.

\section{Température et résistance de la bobine à la fin d'un} tir.

Quand l'impulsion de champ magnétique arrive à extinction toute l'énergie du banc de condensateurs est dissipée en chaleur. Négligeons la résistance des

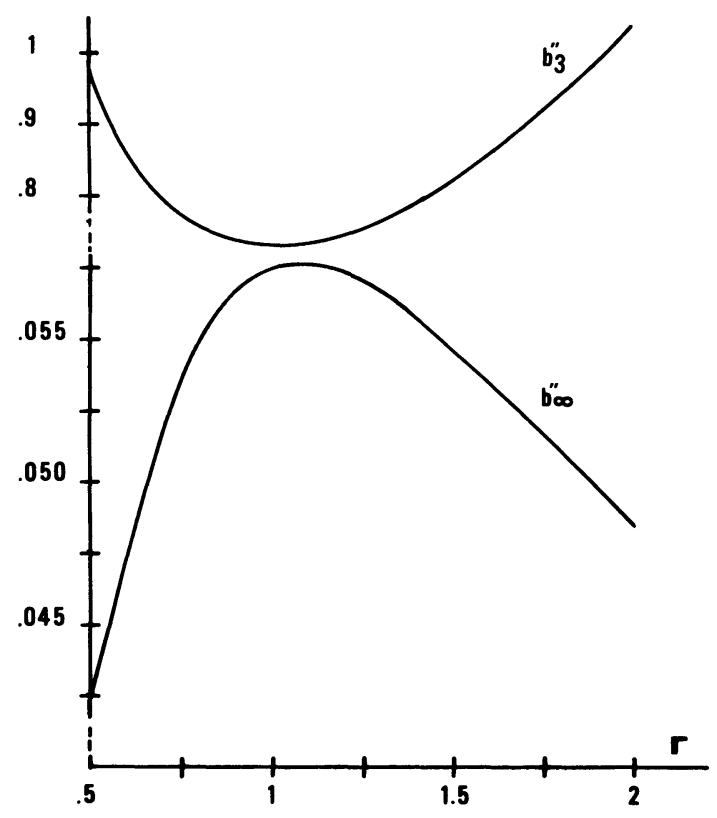

Fig. 6. $-\Delta=\frac{\Gamma^{2}}{w^{2 / 3}} \frac{b_{\infty}^{\prime \prime}}{1-3,5 A_{1} / w^{1 / 3}+b_{3}^{\prime \prime} / w}$.

Pour $A_{1}$ : Voir figure 3.

[For $A_{1}$ : see figure 3.]

éléments du circuit. Dans ces conditions, toute l'énergie joule se trouve dans le cuivre du bobinage, car la frette maintient la bobine adiabatique pendant le court laps de temps que dure le champ pulsé.

Caractérisons l'échauffement du cuivre par la densité de chaleur $Q$ :

$$
Q=\frac{\text { énergie du banc de condensateurs }}{\text { volume du cuivre du bobinage }} .
$$

La définition $\mathrm{du}$ rendement magnétique $\mathcal{R}$, que nous donnons au paragraphe suivant implique 
$1 / 2 C V^{2}=w / \mathcal{R}^{2}$. Par conséquent nous écrivons $Q$, à partir de (1.1), avec pour unité des $\mathrm{J} / \mathrm{cm}^{3}$, sous la forme :

$$
Q=\frac{1,25 B_{0}^{2} w \Gamma}{\pi \lambda \alpha^{3}\left(1-\frac{1}{\alpha^{2}}\right) \mathcal{R}^{2}}=\frac{B_{0}^{2}}{\lambda \mathcal{R}^{2}} \varepsilon(w, \Gamma) .
$$

Nous proposons pour $\varepsilon$ un développement précis, qui est le suivant :

$$
\varepsilon=\frac{\varepsilon_{\infty}}{1-\frac{3 A_{1}}{w^{1 / 3}}-\frac{\varepsilon_{2}}{w^{2 / 3}}+\frac{\varepsilon_{3}}{w}}
$$

$\varepsilon_{\infty}=1,25 \Gamma / \pi A_{\infty}^{3}, \varepsilon_{2}=A_{\infty}^{-2}+3 / 4 A_{1}^{2}$ et $\varepsilon_{3}$ se trouvent décrits sur la figure 7 et des valeurs sont portées sur la table I.

$\varepsilon_{\infty}$, présente un minimum assez plat, puisque dans la plage $0,4<\Gamma<0,9, \varepsilon_{\infty}$ est à moins de $1 \%$ audessus de sa valeur minimale qui est 0,0597 pour $\Gamma=0,64$. Au-delà de $\Gamma=0,9, \varepsilon_{\infty}$ croît lentement pour atteindre 0,0688 à $\Gamma=2$. $\varepsilon$ dépend donc peu du facteur de forme $\Gamma$. Par contre $\varepsilon$ est sensible à $w$. En effet, pour une bobine par exemple carrée, $\Gamma=1$, et peu épaisse $w=5, \varepsilon$ est 3,6 fois la valeur asymptotique $\varepsilon_{\infty}$; par contre pour une bobine de même forme extérieure, mais six fois plus épaisse, $\varepsilon<1,2 \varepsilon_{\infty}$.

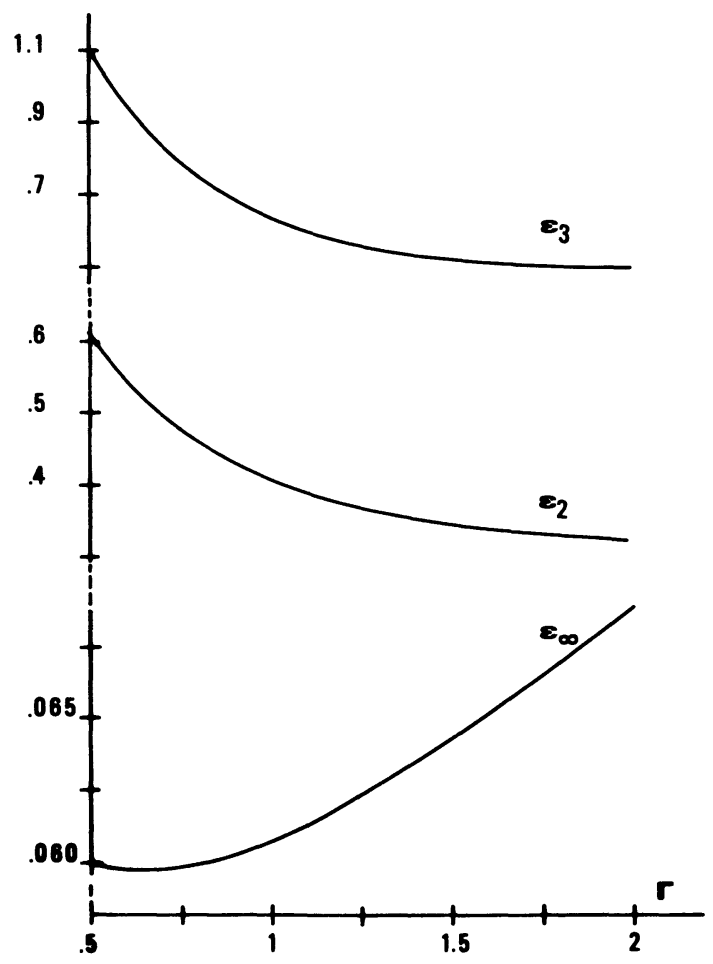

Fig. 7. $-\varepsilon=\frac{\varepsilon_{\infty}}{1-3 A_{1} / w^{1 / 3}-\varepsilon_{2} / w^{2 / 3}+\varepsilon_{3} / w}$. Pour $A_{1}$ : Voir figure 3.

[For $A_{1}:$ see figure 3.]
Cette comparaison souligne tout l'intérêt des bobines épaisses.

Avant le tir suivant, le bobinage doit reprendre sa température cryogénique initiale. Cela demandera un temps de refroidissement d'autant plus long que $Q$ est élevée. Il faut aussi souligner qu'il est impératif que cette température soit bien uniforme, sinon la bobine risque d'être détruite. En effet, supposons localement, une température, juste au début d'un tir, supérieure au bain cryogénique. La résistivité à cet endroit est plus élevée, donc la puissance joule subira en ce point un effet d'emballement qui se traduira par une densité de chaleur apte à brûler l'isolant ou pire, suffisante pour fondre le cuivre.

Il est par conséquent impératif de veiller à une remise en froid correcte du bobinage, ce qui limite la fréquence journalière des tirs. Par exemple, après un tir à $40 \mathrm{~T}$, sur une bobine de 10 litres, épaisse et frettée, l'expérience montre que ce refroidissement demande de $3 / 4$ d'heure à 1 heure.

Les courbes $T(Q)$ et $R(Q) / R_{77}$ que nous avons représentées sur les figures 8 et 9 , donnent, pour la 8 , la température en fin de tir (en Kelvin) et pour la 9 la résistance réduite de la bobine chaude. $T(Q)$ est déduite de l'enthalpie du cuivre [14] et $R(Q) / R_{77}$ correspond au cuivre électrolytique industriel.

Il est raisonnable d'admettre que $200 \mathrm{~K}$ est une limite à ne pas dépasser pour l'échauffement $(Q=$ $327 \mathrm{~J} / \mathrm{cm}^{3}$ ) de la bobine, car déjà à cette température $R_{200} / R_{77}>5$ et la dilatation brutale du cuivre entraîne une pression interne sur le bobinage supérieure à $20 \mathrm{~kg} / \mathrm{mm}^{2}$. Cette limite de $200 \mathrm{~K}$ n'est pas draconnienne, mais elle est l'assurance d'une plus grande longévité des bobines. Nous devons remarquer que $200 \mathrm{~K}$ n'est pas un obstacle au développement ultérieur de champs plus intenses que les actuels, puisque $200 \mathrm{~K}$ correspond à l'échauffement d'une bobine pleine et carrée qui fonctionnerait à plus de $70 \mathrm{~T}$ !

Il y a toujours moyen de réduire $Q$ en plaçant en série avec la bobine une résistance de forte capacité thermique; mais ce serait au détriment de la durée du champ.

Telle que nous l'avons présentée, $Q$ n'est qu'une valeur moyenne prise sur l'ensemble du bobinage. En réalité, par le fait de la magnétorésistance, la distribution de $Q$ est de révolution et son maximum $Q^{*}$ se situe au coeur de la bobine. Par exemple, à $45 \mathrm{~T}$, nous estimons $(\S 9)$ que dans une bobine épaisse qui minimise $Q, Q^{*}$ est majorée de plus de $35 \%$ par rapport à $Q$, ce qui justifie d'autant plus la limite préconisée des $300 \mathrm{~J} / \mathrm{cm}^{3}$ moyens.

\section{Considérations sur le rendement magnétique.}

Définissons le rendement magnétique par $\mathcal{R}=B_{0} / B_{0,0}$ $B_{0}$ est le champ maximum réel, tandis que $B_{0,0}$ représente le champ maximum qui serait obtenu si toute l'énergie du banc de condensateurs était transformée en énergie magnétique. 


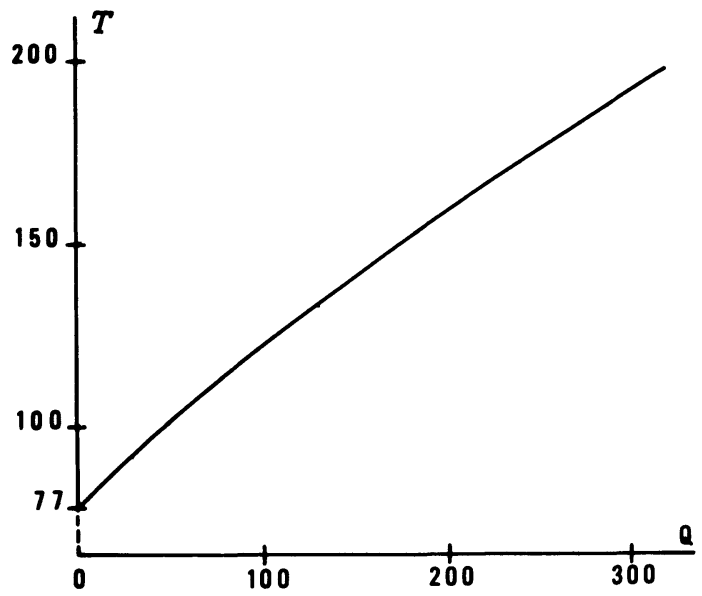

Fig. 8. $-T(Q)$ Température du bobinage à la fin d'un tir. $T$ est exprimée en kelvin et $Q$ en $\mathrm{J} \mathrm{cm}^{-3}$.

$[T(Q)$ is the coil winding temperature after a shot. $T$ is expressed in kelvin and $Q$ in $\mathrm{J} \mathrm{cm}^{-3}$.]

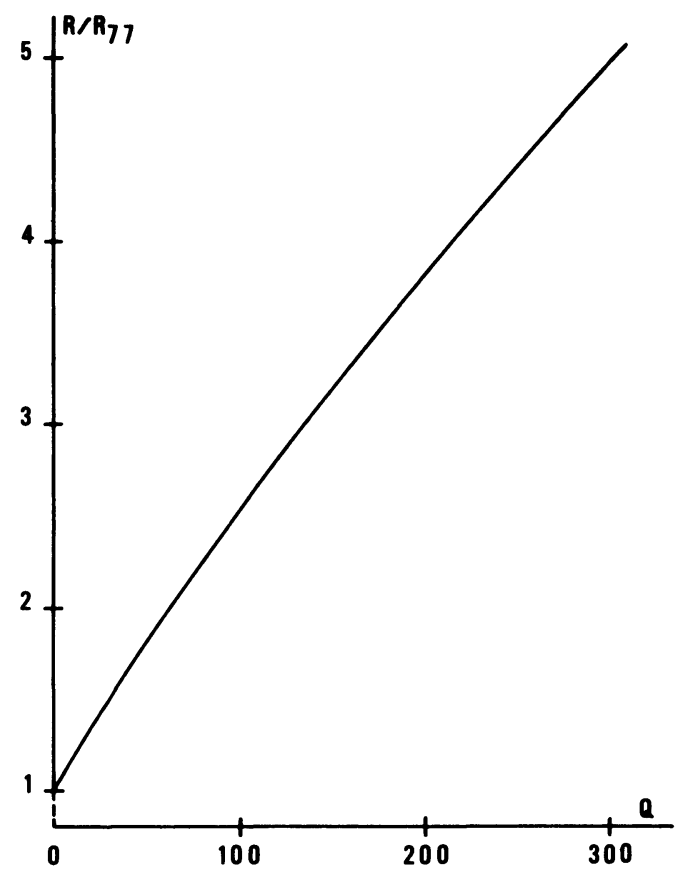

Fig. 9. $-R(Q) / R_{77}$ Résistance réduite de la bobine à la fin d'un tir. $Q$ est exprimé en $\mathrm{J} \mathrm{cm}^{-3}$.

[Value of the coil resistance ratio $R(Q) / R_{77}$, just after a shot. $Q$ units : $\mathrm{J} \mathrm{cm}^{-3}$.]

$\mathbb{R}$ est une donnée économique importante, puisqu'il conditionne l'énergie réelle, $1 / 2 C V^{2}=w / \mathbb{R}^{2}$, qui doit être stockée dans le banc de condensateurs, pour obtenir $\boldsymbol{B}_{\mathbf{0}}$.

Pour déterminer $\mathcal{R}$, nous établirons l'équation qui donne la variation du champ $B(t)$ pendant la phase de décharge des condensateurs vers la bobine. $B$ est produit par le courant $I=I_{0,0} g(t)$; donc $B=B_{0,0} g(t)$.

$g$ est maximum à $t_{\max }$ et $g_{\max }=\mathcal{R}$.
Le courant dans le circuit de la figure 1a, satisfait à l'équation

$$
V+R(t) I+L \frac{\mathrm{d} I}{\mathrm{~d} t}=0
$$

$R(t)$ représente à l'instant $t$ la valeur de la résistance du circuit de la bobine. C'est une fonction croissante du temps à partir de sa valeur initiale $R(0)$ qui est la résistance au début d'un tir. La cause principale de cette croissance est due à l'échauffement adiabatique du bobinage. Cette croissance sera d'autant plus importante que l'est $Q$. L'autre cause de croissance est la magnétorésistance du fil du bobinage. Mais son influence est minime et par conséquent nous la négligerons. Elle est en effet minime car, d'une part quand le champ devient intense la bobine est déjà chaude et l'est de plus en plus, et il en résulte d'après la règle de Kohler [15] que l'accroissement de la résistivité du cuivre réduit la magnétorésistance; et d'autre part cette magnétorésistance n'intervient que là où le champ magnétique est intense, c'est-à-dire sur les spires centrales de la bobine qui ne représentent qu'une faible fraction de la longueur du fil constituant le bobinage, et donc de la résistance de la bobine.

Dérivons (8.1) et introduisons la résistance réduite $r=R(t) / R(0)$. Après le changement de variable $x=$ $\pi t / 2$, faisons apparaître le paramètre d'amortissement $d_{0}^{1 / 2}=t_{0} / \pi(0)$ tel que $\boldsymbol{\tau}(0)=L / R(0)$. Ainsi l'équation différentielle qui gouverne $g(x)$ et qui détermine $\mathcal{R}$ s'écrit :

$$
0=g+2 d_{0}^{1 / 2} g \frac{\mathrm{d} r}{\mathrm{~d} x}+2 d_{0}^{1 / 2} r \frac{\mathrm{d} g}{\mathrm{~d} x}+\frac{\mathrm{d}^{2} g}{\mathrm{~d} x^{2}} .
$$

Il va de soit que $\mathcal{R}$ est une fonction décroissante de $d_{0}^{1 / 2}$ et cette décroissance est amplifiée par $Q$. Justifions cette considération qualitative en exprimant $\mathcal{R}$ à partir du bilan à $t_{\max }$ des énergies non magnétiques qui sont l'énergie joule $\int_{0}^{t_{\max }} g^{2} I_{0,0}^{2} R(t) \mathrm{d} t$ et l'énergie électrostatique non encore utilisée $1 / 2 C I_{0,0}^{2} \mathcal{R}^{2} R^{2}\left(t_{\max }\right)$ car à $t_{\text {max }}$, la tension aux bornes du banc de condensateurs est d'après (8.1): $R\left(t_{\max }\right) I_{0,0} R$; ce qui fait que

$$
\mathcal{R}^{2}=\frac{1-4 d_{0}^{1 / 2} \int_{0}^{x_{\max }} r g^{2} \mathrm{~d} x}{1+4 d_{0} r^{2}\left(x_{\max }\right)} .
$$

Dans un tir à bas champ magnétique $Q$ est négligeable. Disons qu'elle est nulle; ce qui implique que $r$ conserve sa valeur initiale unité pendant tout le tir et (8.2) est réduit à la forme très classique :

$$
0=g+2 d_{0}^{1 / 2} \frac{\mathrm{d} g}{\mathrm{~d} x}+\frac{\mathrm{d}^{2} g}{\mathrm{~d} x^{2}}
$$

dont la solution est

$$
g_{0}(x)=\frac{\sin \left(1-d_{0}\right)^{1 / 2} x}{\left(1-d_{0}\right)^{1 / 2}} \exp -d_{0}^{1 / 2} x .
$$


$\mathcal{R}_{0}\left(d_{0}^{1 / 2}\right)$ est décrit par K. S. W. Champion [10] dans une table de valeurs numériques approximatives, et par B. Montgomery [16] à travers une courbe et aussi une formule relativement lourde. Il est plus simple d'écrire que $g_{0}$ est maximum pour $x_{\max , 0}=$ $\pi t_{\max , 0} / 2 t_{0}$ défini par la relation :

$$
\begin{aligned}
x_{\max , 0}=\frac{\operatorname{Arc} \cos d_{0}^{1 / 2}}{\left(1-d_{0}\right)^{1 / 2}}=\frac{\pi}{2} & -d_{0}^{1 / 2}+ \\
& +\frac{\pi}{4} d_{0}-\frac{2}{z} d_{0}^{3 / 2} \ldots
\end{aligned}
$$

ce qui donne :

$$
\begin{aligned}
\mathcal{R}_{0}=\exp -d_{0}^{1 / 2} x_{\max , 0} & =1-\frac{\pi}{2} d_{0}^{1 / 2}+ \\
+ & 2,234 d_{0}-3 d_{0}^{3 / 2} \ldots
\end{aligned}
$$

Retenons que pour $d_{0}^{1 / 2}=0,1, \Re_{0}=0,86$ et $t_{\max , 0}=$ $0,94 t_{0}=0,3 \pi(0)$.

Dans un tir à fort champ $Q$ peut dépasser dans le cuivre les $300 \mathrm{~J} / \mathrm{cm}^{3}$ (qui est la limite conseillée, à ne pas dépasser) et qui donne, rappelons-le, une résistance $R(Q)$ en fin de tir 5 fois supérieure à sa résistance initiale. Nous avons dit que $Q$ pénalise le rendement magnétique. Une résistance dans le circuit diminuerait $Q$ dans la bobine; mais nous pourrions montrer qu'en fin de tir, la résistance globale est toujours supérieure à $R(Q)$, tandis que le rendement magnétique et le temps de décroissance crowbar seront toujours inférieurs à ceux du circuit dont la résistance est négligeable devant celle de la bobine.

C'est pourquoi nous limiterons l'étude de $\mathcal{R}_{Q}\left(d_{0}^{1 / 2}\right)$ au cas d'un circuit de champ pulsé dit «idéal », où la résistance de la bobine domine largement celle du circuit.

Pour résoudre (8.2), explicitons en premier $r(x)$ en remarquant que l'expression différentielle de la densité de la chaleur $q(x)$ accumulée dans le cuivre depuis le début du tir jusqu'à $x$, s'écrit :

$$
\begin{aligned}
\mathrm{d} q & =\frac{R(0)}{\text { Volume du cuivre }} I_{0,0}^{2} g^{2} r \mathrm{~d} t \\
& =4 d_{0}^{1 / 2} Q g^{2} r \mathrm{~d} x .
\end{aligned}
$$

Ce qui fait que $q(x)$ satisfait à l'équation intégrale :

$$
\int_{0}^{q(x)} \frac{\mathrm{d} q}{r(q)}=4 d_{0}^{1 / 2} Q \int_{0}^{x} g^{2} \mathrm{~d} x .
$$

Tant que $r(q)$ reste inférieur à 3 , la courbe de la figure 9 peut être représentée analytiquement par :

$$
r=1+a q+b q^{2}=1+q / 59-q^{2} / 70000
$$

et cette forme sera utilisée pour résoudre $(8.5 \mathrm{~b})$ car $a$ posteriori nous vérifions, comme le montre la figure 10, que dans tous les cas, du moins pour $Q<400$, $r\left(x_{\max , Q}\right)<3$.

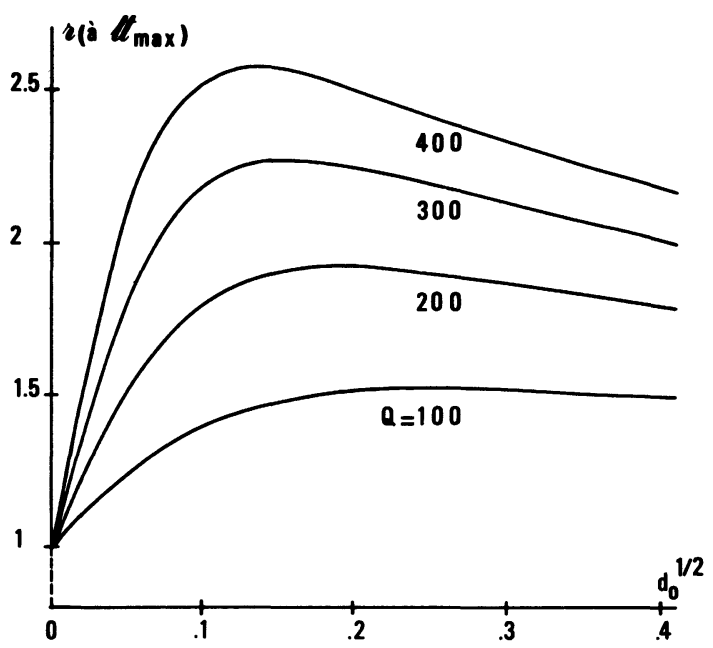

Fig. 10. - Courbes iso- $Q$, de la valeur $r$, de la résistance réduite de la bobine, prise à l'instant où le champ magnétique est maximum. Le circuit considéré est celui d'un champ pulsé " idéal », et la température initiale de la bobine est l'azote liquide.

[Iso- $Q$ curves of the resistance ratio $r$, taken at the time when the magnetic field is maximum. We suppose an «ideal» pulsed magnet electrical circuit and the initial temperature of the coil is liquid nitrogen.]

\section{D’après [17],}

$$
\int \frac{\mathrm{d} y}{1+a y+b y^{2}}=\frac{1}{(a-4 b)^{1 / 2}} \ln \frac{2 b y+a-\sqrt{a^{2}-4 b}}{2 b y+a+\sqrt{a^{2}-4 b}} .
$$

Par conséquent (8.5b) prendra la forme suivante :

$$
4 d_{0}^{1 / 2} Q \sqrt{a^{2}-4 b} \int_{0}^{x} g^{2} \mathrm{~d} x=\ln \frac{2+q\left(a+\sqrt{a^{2}-4 b}\right)}{2+q\left(a-\sqrt{a^{2}-4 b}\right)} .
$$

Inversons la fonction ln dans (8.7) et posons pour alléger l'écriture

$$
u=\exp \left(4 d_{0}^{1 / 2} Q \sqrt{a^{2}-4 b} \int_{0}^{x} g^{2} \mathrm{~d} x\right)
$$

d'où

$$
q=\frac{2}{a+\sqrt{a^{2}-4 b}} \frac{u-1}{1-u \frac{a-\sqrt{a^{2}-4 b}}{a+\sqrt{a^{2}-4 b}}}
$$

qu'il suffit de reporter dans (8.6) pour obtenir $r(x)$. Sa dérivée est immédiate, donc l'équation intégrodifférentielle (8.2) sera explicitée en arrondissant ses paramètres numériques, par l'expression : 


$$
\begin{aligned}
0=g+\frac{\mathrm{d}^{2} g}{\mathrm{~d} x^{2}}+d_{0}^{1 / 2} \frac{\mathrm{d} g}{\mathrm{~d} x}\left\{2+\frac{1,909(u-1)}{1+0,0453 u}\right. & \left.-\frac{0,0906(u-1)^{2}}{(1+0,0453 u)^{2}}\right\}+ \\
+\frac{d_{0} Q}{13,47} & \frac{g^{3} u}{1+0,0453 u}\left\{1,909-\frac{0,268(u-1)}{1+0,0453 u}+\frac{0,0082(u-1)^{2}}{(1+0,0453 u)^{2}}\right\} \\
u & =\exp \frac{d_{0}^{1 / 2} Q}{13,47} \int_{0}^{x} g^{2} \mathrm{~d} x .
\end{aligned}
$$

Prenons en compte (8.3) et (8.8). Il apparaît alors que pour $d_{0}^{1 / 2}$,

$$
\begin{gathered}
\frac{\mathcal{R}_{0}-\mathcal{R}_{Q}}{d_{0} Q}=F_{0}+d_{0}^{1 / 2} Q F_{1}+d_{0} Q^{2} F_{2}+\cdots+d_{0}^{i / 2} Q^{i} F_{i} \cdots \\
\frac{t_{\max , 0}-t_{\max , Q}}{d_{0} Q_{0}}=M_{0}+d_{0}^{1 / 2} Q M_{1}+\cdots+d_{0}^{i / 2} Q^{i} M_{i} \cdots
\end{gathered}
$$

où les $F_{i}$ et les $M_{i}$ ne sont que des fonctions de $d_{0}^{1 / 2}$.

$\mathcal{R}_{Q}$ et $t_{\max , Q} / t_{0}$ sont représentés respectivement sur les figures 11 et 12 par des courbes iso $Q(Q=0$, $100,200,300$ et 400$)$ en fonction de $d_{0}^{1 / 2}$ jusqu'à $d_{0}^{1 / 2}=0,4$, où pour $Q=400, t_{\max }=0,48 \quad t_{0}$ et $\mathcal{R}$ n'est plus que $45 \%$, ce qui signifie que seulement $20 \%$ de l'énergie du banc de condensateurs correspond à l'énergie magnétique $W$. Ces courbes montrent l'importance de $Q$ dans la dégradation de $\mathcal{R}$ et $t_{\max }$, et

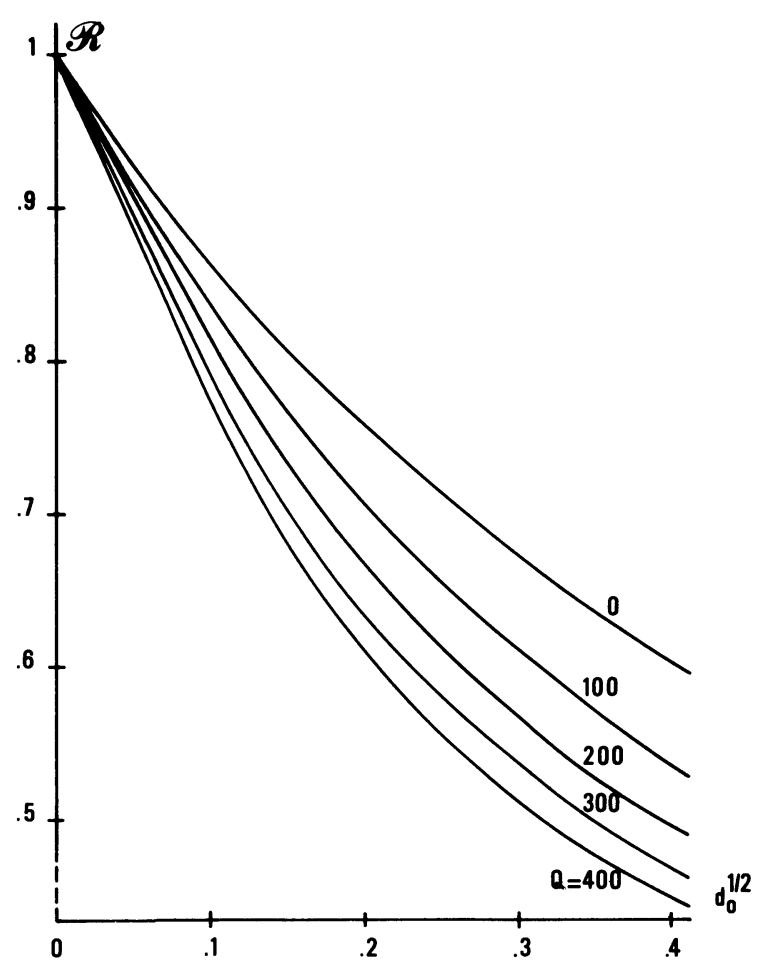

Fig. 11. - Courbes iso- $Q$, du rendement magnétique, calculé dans le cas d'un circuit de champ pulsé « idéal ", avec une bobine plongée dans l'azote liquide.

[Iso- $Q$ curves of the magnetic conversion efficiency $\mathcal{R}$ calculated for the case of an "ideal » pulsed magnet generator with the coil immersed in liquid nitrogen.] soulignent par là-même, l'intérêt des bobines épaisses qui minimisent $Q$.

Le propre d'un champ pulsé crowbar, quasistatique, est de privilégier la qualité du transfert de l'énergie stockée, en énergie magnétique. Toutefois, nous devons rejeter l'idée d'un $R$ unité, pour des raisons économiques, techniques et expérimentales. En effet, un tel $\mathcal{R}$ implique $d_{0}^{1 / 2}$ quasiment nul, c'est-àdire un transfert de l'énergie très rapide, ce qui sous-

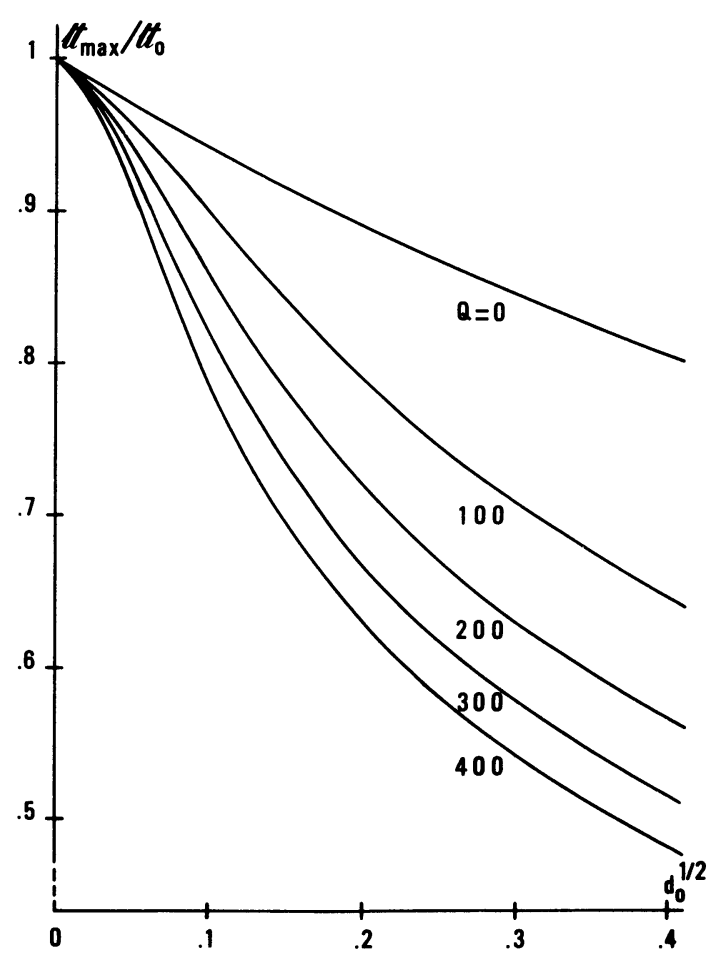

Fig. 12. - Courbes iso- $Q$ du rapport $\pi^{\max } t_{0}$ calculées dans le cas d'un circuit de champ pulsé "idéal ", avec une bobine plongée dans l'azote liquide.

[Iso- $Q$ curves of the ratio $\pi^{\max } / t_{0}$, calculated for the case of an "ideal " pulsed magnetic generator with the coil immersed in liquid nitrogen.] 
entend des courants très intenses, donc l'utilisation de condensateurs à décharge rapide, hors de prix. Pour satisfaire à $u_{0}$ très court, l'inductance de la bobine doit être très faible ce qui est difficilement réalisable car le fil de bobinage doit avoir une section importante; de plus une telle bobine offrirait une très faible résistance qui serait alors bien inférieure à celle du circuit de décharge. Enfin, une décharge rapide induit un signal parasite sur les échantillons ce qui est préjudiciable aux mesures.

$d_{0}^{1 / 2}$ est donc un compromis qui ne doit raisonnablement pas dépasser 0,1 ; car pour cette valeur et $Q=400$, l'énergie magnétique représente déjà moins de $60 \%$ de l'énergie du banc.

Le rendement magnétique déduit de la figure 11 est insuffisamment précis pour établir un projet. Par conséquent, et avec une précision plus que nécessaire nous représenterons $(8.9 \mathrm{a})$ et $(8.9 \mathrm{~b})$ dans la gamme $0-0,1$ des $d_{0}^{1 / 2}$ par :

$$
\mathcal{R}_{0}-\mathcal{R}_{Q}=d_{0} Q\left(0,0418-0,237 d_{0}^{1 / 2}+0,57 d_{0}\right)(1+f)
$$

$$
\begin{aligned}
t_{\max , 0}- & \boldsymbol{t}_{\max , Q}= \\
& =d_{0} Q_{0}\left(0,0593-0,241 d_{0}^{1 / 2}+0,47 d_{0}\right)(1+m) .
\end{aligned}
$$

Les fonctions correctrices $f\left(Q, d_{0}^{1 / 2}\right)$ et $m\left(Q, d_{0}^{1 / 2}\right)$ seront déduites par une interpolation linéaire, respectivement des courbes iso- $Q$ des figures 13 et 14 .

\section{Appendice. Un exemple concret.}

Que seront, en fonction de $t_{\max }$ et pour le champ pulsé $B_{0}=45 \mathrm{~T}-W=1 \mathrm{MJ}-2 a_{1}=1,4 \mathrm{~cm}$, l'énergie $1 / 2 \mathrm{CV}^{2}$ et la résistance du bobinage à la fin d'un tir ?

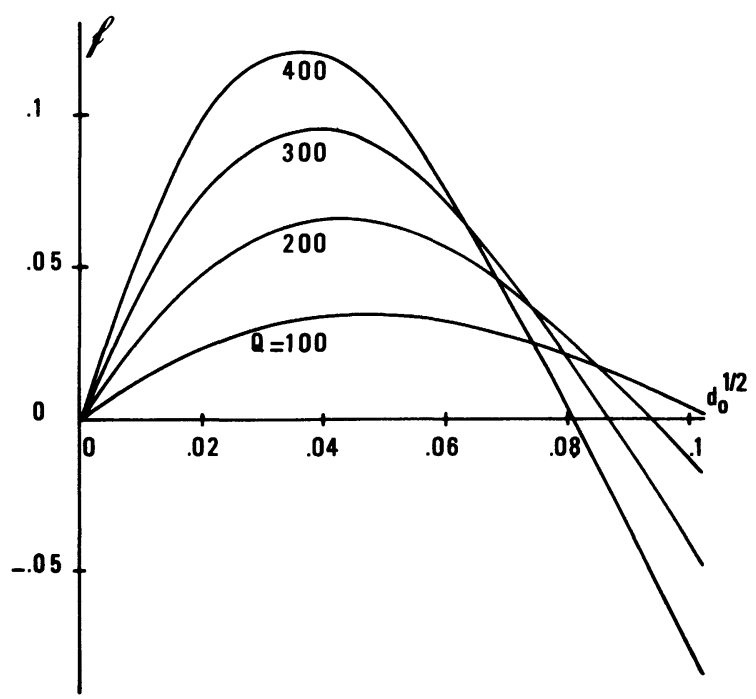

Fig. 13. - Courbe iso- $Q$ de la fonction correctrice $f$.

[Iso- $Q$ curves of the correcting factor $f$.]

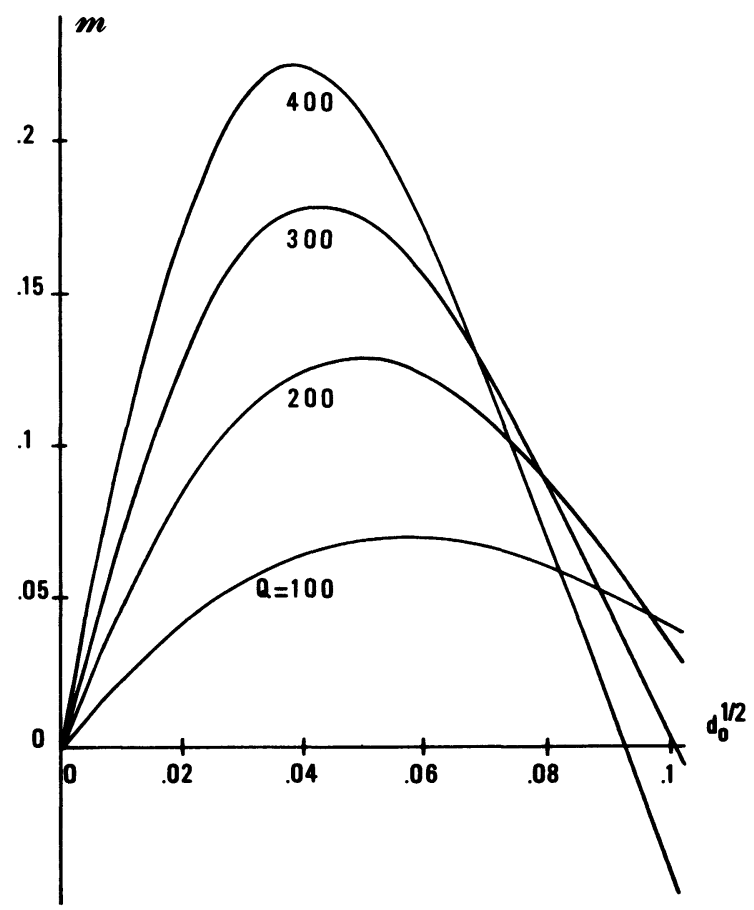

Fig. 14. - Courbe iso- $Q$, de la fonction correctrice $m$.

[Iso- $Q$ curves of the correcting factor $m$.]

Ce champ pulsé est celui dont il est fait mention dans l'introduction. Nous choisirons une bobine carrée, $\Gamma=1$, car cette géométrie est un bon compromis qui minimise à la fois $Q$ et la densité de courant dans le bobinage.

A partir de (1.1), puis de (3.8), (4.2), (6.4) nous obtenons respectivement les caractéristiques suivantes : $w=575,9,2 a_{2}=20,7 \mathrm{~cm}, \pi=0,72 \mathrm{~s}$ à l'azote liquide et pour un coefficient de remplissage $\lambda=0,9$; enfin l'homogénéité du champ est $5 \times 10^{-4}$ dans une sphère centrée de $1 \mathrm{~cm}$ de diamètre.

D'après (7.1) à $W$ correspond $Q_{0}=161 \mathrm{~J} / \mathrm{cm}^{3}$. Introduisons dans $(8.10 a) Q=Q_{0} / \mathcal{R}^{2}$ et résolvons $\mathcal{R}_{Q}\left(d_{0}^{1 / 2}\right)$; puis à l'aide de $(8.10 \mathrm{~b}), t_{\max , Q}\left(d_{0}^{1 / 2}\right) .1 / 2^{\circ} C V^{2}$ est à $10^{6} \mathrm{~J}$ ce que $Q$ est à $Q_{0}$. Ainsi nous pouvons représenter dans la figure $151 / 2 C V^{2}$ en fonction de $t_{\max }$. Cette courbe montre un nécessaire supplément de $15 \%$ d'énergie pour $t_{\max }=85 \mathrm{~ms}$ et $54 \%$ pour $190 \mathrm{~ms}$ !

La valeur de la résistance réduite de la bobine à la fin d'un tir $r\left(Q=R(Q) / R_{77}\right.$ est estimée en utilisant la courbe de la figure $9 . r(Q)$ est représentée en fonction du choix de $t_{\max }$, sur la figure 16. Egal à 3,33 pour $W$, $r$ croît avec $t_{\max }$, ce qui réduit le temps crowbar. Il prend la valeur 3,67 pour $t_{\max }=85 \mathrm{~ms}$ et atteint 4,38 lorsque $t_{\max }=190 \mathrm{~ms}$.

Au coeur de la bobine, dans les spires centrales, à cause de la magnétorésistance, la densité de chaleur $Q^{*}$ accumulée dans le cuivre pendant tout un tir est supérieure à $Q$. Il en est de même, là, de la résistivité locale du cuivre que nous traduirons par le rapport $r^{*}\left(Q^{*}\right)$, qui la compare à la résistivité à l'azote liquide. 


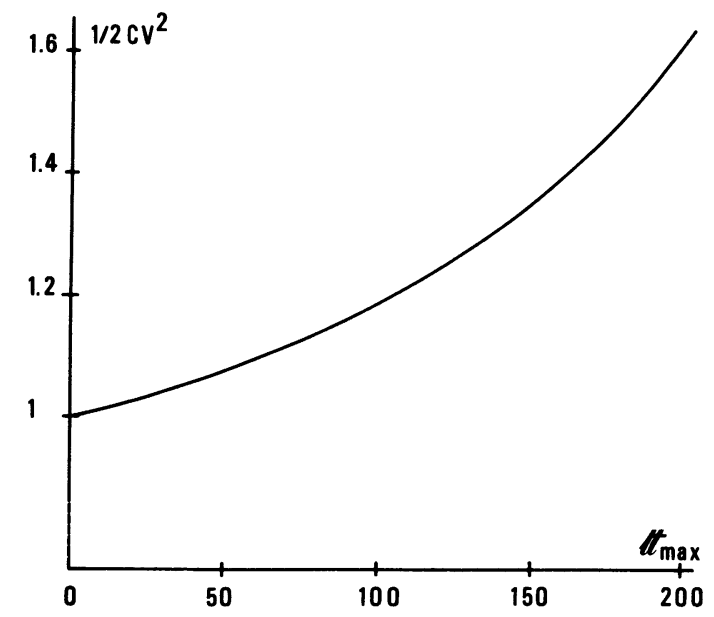

Fig. 15. - Cas d'un champ pulsé crowbar «idéal " $B_{0}=$ $45 \mathrm{~T} ; W=10^{6} \mathrm{~J} ; 2 a_{1}=1,4 \mathrm{~cm}$ avec une bobine carrée dans l'azote liquide. Valeur suivant $t^{\max }$ de l'énergie qui doit être stockée dans le banc de condensateurs pour obtenir $45 \mathrm{~T}$. $1 / 2 C V^{2}$ est exprimée en $\mathrm{MJ}$ et $t^{\max }$ en $\mathrm{ms}$.

[Values as a function of $t^{\max }$ of the energy to be stored in the condensers for obtaining $45 \mathrm{~T}$ in the case of the "ideal " pulsed magnetic field defined by $B_{0}=45 \mathrm{~T} ; W=1 \mathrm{MJ}$; $2 a_{1}=1,4 \mathrm{~cm} ; \Gamma=1$. The coil is immersed in liquid nitrogen. $1 / 2 C V^{2}$ units : $10^{6} \mathrm{~J}$. $\sharp^{\max }$ units : $\mathrm{ms}$.]

En vue d'établir $Q^{*}$, nous constatons que la magnétorésistance transverse du cuivre électrolytique industriel est bien représentée à l'azote liquide (du moins jusqu'à $50 \mathrm{~T}$ ) par la forme analytique suivante :

$$
\Delta \rho / \rho_{77}=0,01752 B-0,14717(1-\exp -0,0985 B) .
$$

C'est dire, d'après la règle de Kohler [15], quand le rapport de résistivité est $r^{*}$, que le champ apparent est $B^{*}=B / r^{*}$. En conséquence la magnétorésistance s'écrit :

$$
\Delta \rho / \rho=0,01752 B^{*}-0,14717\left(1-\exp -0,0985 B^{*}\right) .
$$

Il s'ensuit que $Q^{*}$ est la solution asymptotique de l'équation différentielle suivante, qui est déduite de (8.5a), soit :

$$
\frac{\mathrm{d} q^{*}}{\mathrm{~d} x}=4 d_{0}^{1 / 2} Q g^{2} r^{*}\left(q^{*}\right)\left\{1+\frac{\Delta \rho\left(B^{*}\right)}{\rho}\right\} .
$$

Compte tenu que la magnétorésistance modifie peu l'impulsion de champ magnétique, compte tenu que la formule (8.6) qui représente $r(q)$ jusqu'à $q=140$, n'introduit au-delà et jusqu'à $q=250$ qu'une erreur au plus de $1 \% ; g(x)$ sera d'abord solution de l'équation intégrodifférentielle (8.8) jusqu'à ce qu'apparaisse la phase de décroissance crowbar, c'est-à-dire à partir de $x_{0}$ tel que pour cette valeur, $g^{\prime}+2 d_{0}^{1 / 2} r g=0$. $x_{0}$ est légèrement supérieur à $\pi / 2\left(x_{0}-\pi / 2 \simeq \pi / 2-x_{\max }\right)$.

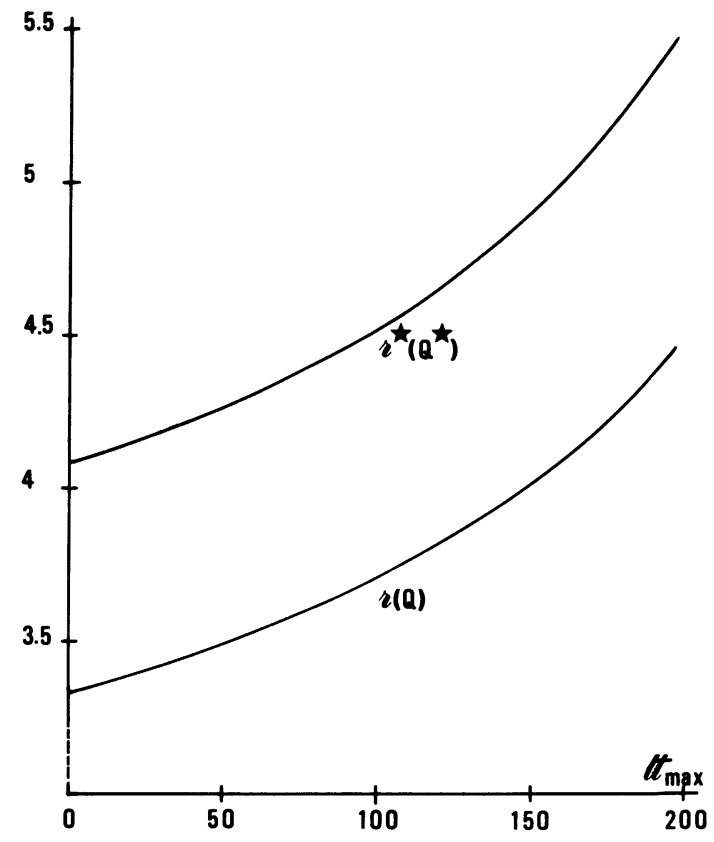

Fig. 16. - Cas d'un champ pulsé crowbar «idéal » $B_{0}=$ $45 \mathrm{~T} ; W=10^{6} \mathrm{~J} ; 2 a_{1}=1,4 \mathrm{~cm}$ avec une bobine carrée dans l'azote liquide. Valeurs suivant $t^{\max }$, de la résistance réduite de la bobine à la fin d'un tir et de la résistivité réduite du cuivre dans les spires centrales, $r^{*}\left(Q^{*}\right)$. $t^{\max }$ est exprimé en ms.

[For the « ideal " pulsed magnetic field $B_{0}=45 \mathrm{~T} ; W=$ $1 \mathrm{MJ} ; 2 a_{1}=1,4 \mathrm{~cm} ; \Gamma=1$ with a coil immersed in liquid nitrogen. Values as a function of $\mathrm{tt}^{\max }$ of the coil resistance ratio $r(Q)$ at the end of a shot, and at the same time the copper resistivity ratio of the inner winding turns $r^{*}\left(Q^{*}\right)$. $\mathbb{t}^{\max }$ units : ms.

Ensuite, au-delà de $x_{0}, g$ est solution de l'équation intégrodifférentielle

$$
0=g^{\prime}+2 d_{0}^{1 / 2} r g .
$$

Quant à $r^{*}\left(q^{*}\right)$, puisque à $t_{\max }=190 \mathrm{~ms}, Q^{*}$ sera certainement largement supérieur à 250 , nous le représenterons par le développement polynomial suivant $1+\frac{q^{*}}{58,07}-\frac{q^{* 2} \times 10^{-4}}{5,285}+\frac{q^{* 3} \times 10^{-6}}{52,52}$ qui est encore valable au-delà de 400 .

Enfin, nous écrivons $B^{*}=\frac{45 g}{r^{*} g_{\max }}$ puisque $B_{0}=$ $45 \mathrm{~T}$.

La résolution simultanée de $g(x)$ et de (9.2), donne pour $x$ infini $Q^{*} / Q . Q^{*} / Q$ décroît très lentement et presque linéairement avec $t_{\max }$. Sa valeur qui est 1,384 pour 0 , passe par 1,369 pour $t_{\max }=85 \mathrm{~ms}$ et atteint 1,351 à $t_{\max }=190 \mathrm{~ms}$.

Nous représentons sur la figure $16 r^{*}\left(Q^{*}\right)$ en fonction de $t_{\max }$. Egal à 4,09 pour $W$, il va de soi que $r^{*}\left(Q^{*}\right)$ croît avec $t_{\max }$. Sa valeur passe par 4,44 pour $t_{\max }=85 \mathrm{~ms}$ et atteint 5,37 pour $190 \mathrm{~ms}$. 
$\mathrm{Vu}$ les figures 15 et 16 , un choix de $t_{\max }$, voisin de $85 \mathrm{~ms}$ est raisonnable. C'est ainsi que fut réalisé, au Service CNRS des Champs Intenses de Toulouse, ce champ quasi-statique de $45 \mathrm{~T}$, en utilisant un banc de condensateurs de $25000 \mu \mathrm{F}$.

Le fil choisi est un fil rectangulaire, $2,8 \times 3,15 \mathrm{~mm}^{2}$, de rayon d'arrondi $0,5 \mathrm{~mm}$ (section $8,61 \mathrm{~mm}^{2}$, ancienne norme UTE C31-211). Compte tenu d'un coefficient de remplissage de 0,9 dû au vernis d'isolement et au collage des spires, nous obtenons $t_{0}$ à partir de (5.2), et nous déduisons à $45 \mathrm{~T}, t_{\max }=83 \mathrm{~ms}$ et $I_{\max }=$ $4000 \mathrm{~A}$.

\section{Bibliographie}

[1.a] Panel on high magnetic field, Research and Facilities, Solid State Sciences Committee (1979), U.S.A. National Research Council.

[1.b] Miura, N. and Herlach, F., Strong and Ultrastrong Magnetic Fields, ed. F. Herlach, Topics in Applied Physics, Vol. 57 (Springer-Verlag, Heidelberg) 1985.

[2] Askenazy, S., Proceedings of the MFSP Conference, Oxford 1978, 101.

[3] Leupold, M. J., Hale, J. R., Iwassa, Y., Rubin, L. G., WegGel, R. J., IEEE Trans. Mag. MAG 17 (1981) 1966.

[4] Ozhogin, V. I., Gurtovoj, K. G., Lagutin, A. S., Proceedings of the Int. Symposium on High Field Magnetism, Osaka (North-Holland Publishing Company), 1982, 267.

[5] Sultanem, F., Bleus, C. A., Postel, C., Askenazy, S., Marquez, J., 8th Int. Conference on Magnet. Technology, J.. Physique Colloq. 45 (1984) C1-67.

[6] Marquez, J., Ulmet, J. P., Leotin, J., Proceedings Photon 83, 201 (The International Society for Optical Engineering).
[7] Clark, A. F., Childs, G. E., Wallace, G. H., Cryogenics 10 (1970) 295.

[8] Butterworth, Philos. Mag. 29 (1915) 578.

[9] Askenazy, S., 2e partie de cet article, en préparation.

[10] Champion, K. S. W., Proc. Phys. Soc. B 38 (1950) 795.

[11] Grover, F., Sci. paper Bureau Standards 455, 18 (1922) 451.

[12] Dwight, H. B., Electr. World 71 (1918) 301 (formule $\mathrm{n}^{\mathrm{o}} 5$ ).

[13] Durand, E., Magnétostatique (Masson Editeur, Paris) 1968 , p. 270.

[14] Conte, R. R., Eléments de Cryogénie (Masson Editeur, Paris) 1970, p. 272.

[15] KoHler, Ann. Phys. (5) 32 (1938) 211.

[16] Montgomery, D. B., Solenoid Magnet Design (John Wiley and Sonc, New York) 1968, Chap. VII and VIII.

[17] SPIEGel, M. R., Mathematical Handbook of Formulae and tables (McGraw-Hill Inc., New York) 1974, Schaum's out lines Series, p. 71. 